\title{
An overview of measurement comparisons from the INTEX-B/MILAGRO airborne field campaign
}

\author{
M. M. Kleb ${ }^{1}$, G. Chen ${ }^{1}$, J. H. Crawford ${ }^{1}$, F. M. Flocke ${ }^{2}$, and C. C. Brown ${ }^{1,3}$ \\ ${ }^{1}$ NASA Langley Research Center, Hampton, Virginia, USA \\ ${ }^{2}$ National Center for Atmospheric Research, Boulder, Colorado, USA \\ ${ }^{3}$ Science Systems and Applications, Inc., Hampton, Virginia, USA
}

Received: 22 April 2010 - Published in Atmos. Meas. Tech. Discuss.: 18 May 2010

Revised: 18 November 2010 - Accepted: 21 November 2010 - Published: 11 January 2011

\begin{abstract}
As part of the NASA's INTEX-B mission, the NASA DC-8 and NSF C-130 conducted three wing-tip to wing-tip comparison flights. The intercomparison flights sampled a variety of atmospheric conditions (polluted urban, non-polluted, marine boundary layer, clean and polluted free troposphere). These comparisons form a basis to establish data consistency, but also should also be viewed as a continuation of efforts aiming to better understand and reduce measurement differences as identified in earlier field intercomparison exercises. This paper provides a comprehensive overview of 140 intercomparisons of data collected as well as a record of the measurement consistency demonstrated during INTEX-B. It is the primary goal to provide necessary information for the future research to determine if the observations from different INTEX-B platforms/instrument are consistent within the PI reported uncertainties and used in integrated analysis. This paper may also contribute to the formulation strategy for future instrument developments. For interpretation and most effective use of these results, the reader is strongly urged to consult with the instrument principle investigator.
\end{abstract}

\section{Introduction}

The Intercontinental Chemical Transport Experiment-B (INTEX-B) was the second major airborne field mission conducted in the spring of 2006 as part of the NASA-led INTEXNA (North America) mission, aiming to investigate the transport and transformation of pollution over the North American continent. INTEX-B operated in coordination with a larger program, the MILAGRO (Mega-city Initiative: Local and Global Research Observations) and IMPEX (Inter-

Correspondence to: M. M. Kleb

(mary.m.kleb@nasa.gov) continental and Mega-city Pollution Experiment) missions. INTEX-B was comprised of two phases. Phase one occurred from 1-21 March to maximize overlap with the MILAGRO campaign. During this phase, observations were primarily over Mexico and the Gulf of Mexico. The second phase lasted from 15 April to 15 May and focused on Asian pollution transported across the Pacific Ocean. Five specific goals were identified for INTEX-B: (1) to investigate the extent and persistence of the outflow of pollution from Mexico; (2) to understand the transport and evolution of Asian pollution, the related air quality, and climate implications in western North America; (3) to relate atmospheric composition to chemical sources and sinks; (4) to characterize the effects of aerosols on radiation; and (5) to validate satellite observations of tropospheric composition (Singh et al., 2009). For a complete mission overview, reader is referred to Singh et al. (2009).

The INTEX-B field mission involved two comparably equipped aircraft, the NASA DC-8 and NSF C-130. The sampling strategy often required coordination of both aircraft while making measurements in different regions or times. This naturally led to the pre-planning and execution of a series of comprehensive measurement comparisons of species/parameters measured on both platforms. The overarching goal was to generate a program-wide unified data set from all available resources to better address the science objectives. These comparisons form a basis to establish data consistency. The INTEX-B measurement comparison exercise should also be viewed as a continuation of efforts aiming to better understand and reduce measurement differences as identified in earlier field intercomparison exercises (e.g. NASA TRACE-P, Eisele et al., 2003; and ICARTT, http://www-air.larc.nasa.gov/missions/ intexna/meas-comparison.htm). It is recognized that further comparisons of the in-situ data sets to satellite retrievals, lidar, and model output are equally important; however such analyses are beyond the scope of this paper. 


\section{Background}

NASA has a long history of conducting instrument intercomparisons beginning with ground-based intercomparisons in July 1983 (Hoell et al., 1984, 1985a,b; Gregory et al., 1985) prior to the commencement of the airborne field studies in October 1983 with the Chemical Instrumentation Test and Evaluation (CITE) missions (Beck et al., 1987; Hoell et al., 1990, 1993; Gregory et al., 1993a,b,c). These early instrument intercomparisons were conducted on a common aircraft platform and played an important role in understanding the sensitivity of different techniques and evaluating them to find the best possible field instrument. The early intercomparison effort stimulated the development of atmospheric measurement techniques/instruments benefitting airborne field programs to this day. Since early 2000 , integrated field campaigns have made use of the same measurement technique on separate aircraft platforms or different measurement techniques sometimes on the same or separate aircraft platforms. To understand the differences seen in the data and to better utilize the data from various instruments, a careful and thorough intercomparison is needed. The first NASA two-aircraft intercomparison was conducted during the 2001 TRACE-P (Transport and Chemical Evolution over the Pacific) field campaign (Eisele et al., 2003). During TRACE-P the NASA DC-8 and P-3B flew wing-tip to wingtip within $1 \mathrm{~km}$ of each other on three occasions lasting between 30 and $90 \mathrm{~min}$. A significant finding of this exercise was that an intercomparison between two aircraft can reveal important insight into instrument performance. It also verified that two aircraft can be flown in a manner such that both sample the same airmass and experience the same high and low frequency fluctuations necessary to evaluate common measurements. In general the best agreement was achieved for the most abundant species $\left(\mathrm{CO}_{2}\right.$ and $\left.\mathrm{CH}_{4}\right)$ with mixed results for less abundant species and those with shorter lifetimes (Eisele et al., 2003). The TRACE-P comparison of fast $(1 \mathrm{~s})$ measurements for $\mathrm{CO}$ and $\mathrm{O}_{3}$ provided valuable information in defining bulk airmass properties, which was useful in interpreting the comparison results for short-lived species. The effect of small scale spatial variation should not have significant impact on assessment of the systematic difference, especially when the range of comparison is sufficiently larger than these variations.

Following TRACE-P, another major coordinated intercomparison occurred in 2004 during the International Consortium for Atmospheric Research on Transport and Transformation (ICARTT) airborne missions (INTEX-A, NEAQS-ITCT 2004, and ITOP). Five wing tip to wing tip intercomparison flights were conducted allowing comparisons between four aircraft. Although not formally published, these intercomparisons and additional mission information can be found in the Measurement Comparisons: ICARTT/INTEX-A link at http://www-air.larc.nasa. gov/missions/intexna/intexna.htm.
Table 1. Chemical Conditions for Intercomparison Periods.

\begin{tabular}{llr}
\hline Date & Air quality conditions & $\begin{array}{r}\text { CO range } \\
\text { (ppbv) }\end{array}$ \\
\hline 19 March 2006 & $\begin{array}{l}\text { Polluted urban and clean MBL } \\
\text { off coast of Mexico }\end{array}$ & $103-223$ \\
17 April 2006 & $\begin{array}{l}\text { Polluted and clean FT } \\
\text { 15 May 2006 }\end{array}$ & $\begin{array}{l}\text { Clean FT and MBL off CA } \\
\text { and OR coast }\end{array}$ \\
\hline
\end{tabular}

The purpose of this paper is to provide a straightforward and comprehensive overview and record of the measurement consistency as characterized through the analysis of the INTEX-B intercomparison data. This paper is not intended as a review of instrument operation but rather a means to highlight the demonstrated instrument performance during the intercomparison periods. Intercomparison results are intended to identify measurements where an investment in improving measurement capability would be of great benefit. Results are also crucial to ensuring that analysis and modeling activities based on multi-platform observations reach conclusions that can be supported within the assessed data uncertainties. For parties interested in making use of the data presented here, further consultation with the relevant measurement investigators is strongly recommended. The remainder of this paper presents the details of the INTEX-B intercomparison.

Section 3 describes the intercomparison approach and implementation, including a description of the types of comparisons is presented. Data processing procedures and statistical assessment are presented in Sect. 4. four. Section 5 contains the results, and the summary is contained in Sect. 6.

\section{Approach/implementation}

During the INTEX-B/MILAGRO/IMPEX field campaigns, three formal measurement comparisons were carried out on 19 March, 17 April, and 15 May 2006. These segments were well integrated into science flights to achieve the overall science goals while aiming to compare instruments/measurements under a wide variety of conditions as summarized in Table 1. During the intercomparison portion of the flights, aircraft separation was less than $300 \mathrm{~m}$ in the horizontal and less than $100 \mathrm{~m}$ in the vertical. The intercomparison period for the 19 March flight was $41 \mathrm{~min}$ (Fig. 1a), covered altitudes from 0.3 to $3.4 \mathrm{~km}$, and encountered Mexico City pollution as well as marine boundary layer air off the coast of Mexico. The wide range of the chemical conditions is evident in $\mathrm{CO}$ levels observed during the intercomparison period which ranged from 103 to $223 \mathrm{ppbv}$. The 17 April (Fig. 1b) intercomparison period lasted 44 min with conditions ranging from polluted at $3.5 \mathrm{~km}$ over northern 
California to clean at $6 \mathrm{~km}$ over southern Oregon. Again the range in chemical conditions can be inferred from the CO levels encountered ( 99 to 163 ppbv). The last intercomparison flight on 15 May (Fig. 1c) was the longest, lasting approximately $1 \mathrm{~h}$. This intercomparison began in the clean free troposphere (about $5.5 \mathrm{~km}$ ) off the northern Oregon coast and ended in the marine boundary layer (near $0.3 \mathrm{~km}$ ) off the northern California coast. As with the two previous intercomparisons, a variety of chemical conditions existed. For these comparisons, data from all three flights were combined for analysis and only data with values greater than the limit of detection were used for analysis. The comparisons cover short-lived to long-lived gas phase species as well as particulate microphysical, optical, and chemical properties. Table 2 provides a detailed list of the species/parameters included in the intercomparison along with measurement techniques, aircraft platform, principal investigators (PI), measurement uncertainties, and confidence level. All of the above information was taken from the PI file headers except for confidence level. For an explanation of "Technique", the reader is referred to the individual PI files located on the INTEX-B website (http://www-air.larc.nasa. gov/missions/intex-b/intexb.html) under the Current Archive Status link. The reported analysis was based on data submissions prior to 1 January 2010. The online plots may change to reflect the data updates at a later date.

In addition to the uncertainty information provided in the PI file headers, a special effort was made to obtain measurement uncertainties which were not originally provided in the file header as well as confidence levels. This is necessary information to determine if measurements are consistent and important metadata for future analysis. Some reported total uncertainties were given in 1 or 2 sigma confidence level while in other cases, confidence levels were not specified. The confidence level is typically associated with precision or precision dominated uncertainties. In some cases, both precision and accuracy are explicitly given in Table 2, while only total uncertainties are provided by the PI in many other cases without clear association to a confidence level. The concept of confidence level may be ill-defined for cases where accuracy is the dominant component of the total uncertainty. In these cases, the readers are directed to measurement PIs for proper application of the uncertainty information.

It is imperative that both aircraft sample the same airmass during the intercomparison period. In practice, this is conducted by keeping the aircraft in close proximity while maintaining a safe separation. Analysis of the fastest measurements can be an effective way to ensure the same airmass was sampled by both aircraft. If the same airmass is sampled, we expect the large scale features to be captured by both instruments. This is illustrated in the time series plots for both ozone (19 March) and water (15 May) where the major features are well represented by both instruments in each comparison (Figs. 2a and 3a). While the most prominent features are apparent in the data from each instrument, there is less
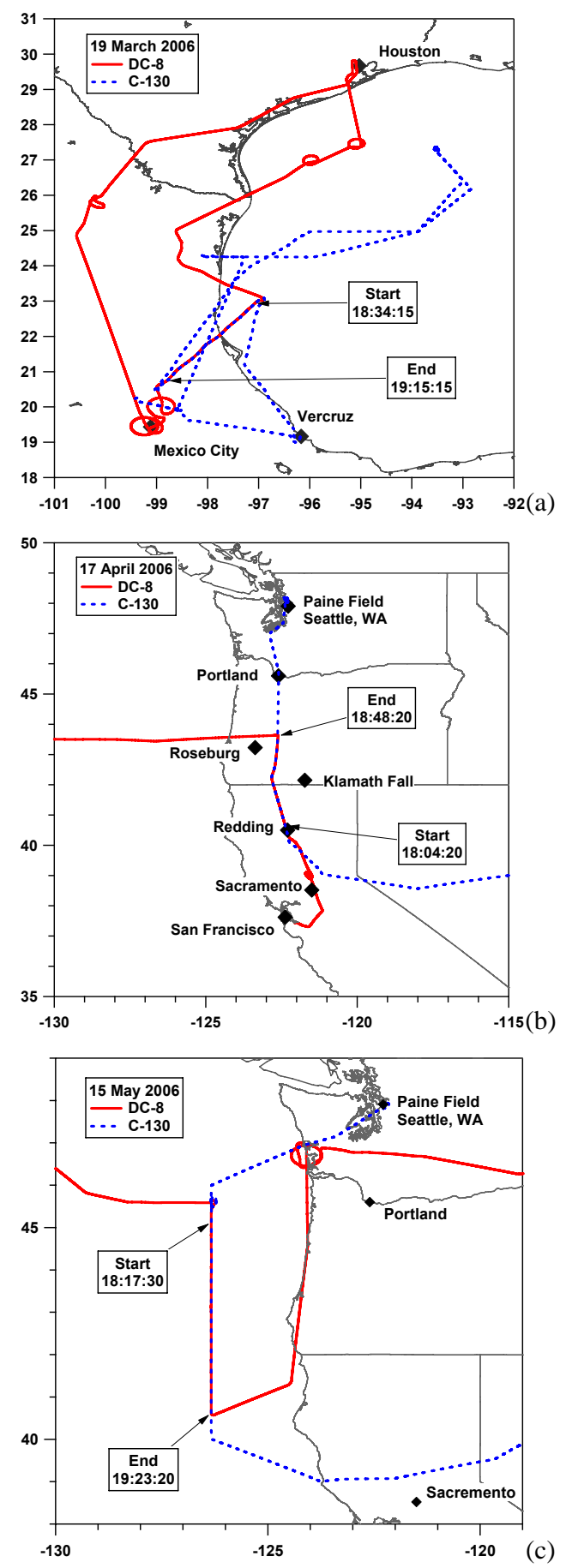

Fig. 1. (a) NASA DC-8 and NSF C-130 flights on 19 March 2006. The intercomparison period is indicated by the start and end times. The DC-8 flight path is shown as a solid red line. The C-130 flight path is shown as a blue dotted line. (b) NASA DC-8 and NSF C-130 flights on 17 April 2006. The intercomparison period is indicated by the start and end times. The DC- 8 flight path is shown as a solid red line. The C-130 flight path is shown as a blue dotted line. (c) NASA DC-8 and NSF C-130 flights on 15 May 2006. The intercomparison period is indicated by the start and end times. The DC-8 flight path is shown as a solid red line. The C-130 flight path is shown as a blue dotted line. 


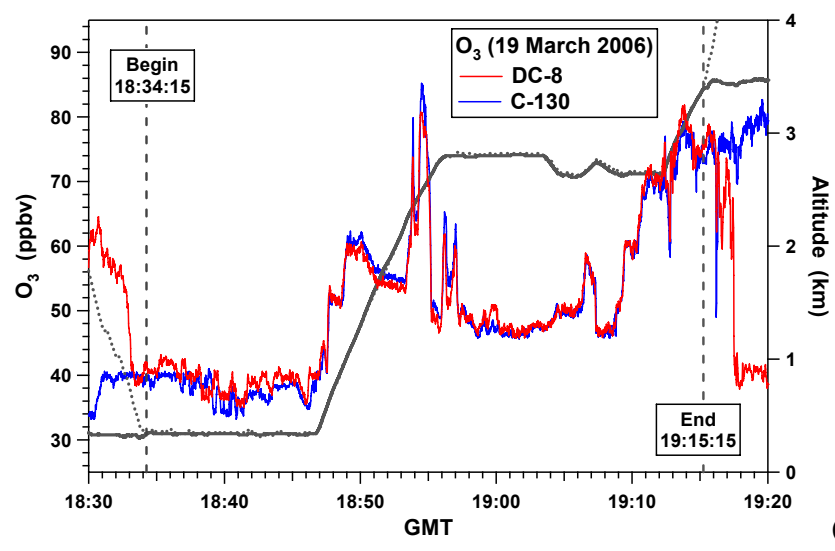

(a)

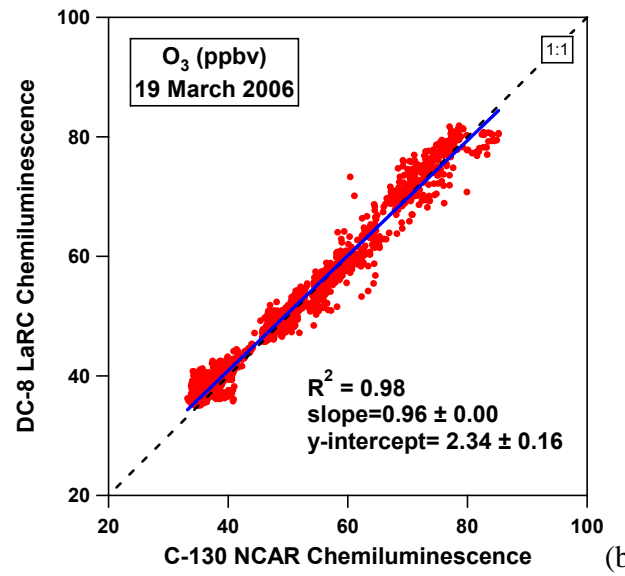

Fig. 2. (a) Timeseries for ozone during the intercomparison portion of the 19 March 2006 flight. The dotted line indicates the DC-8 altitude, solid thick line the C-130 altitude, red line DC-8 ozone, and blue line C-130 ozone. (b) Scatter plot and orthogonal distance regression for the $\mathrm{DC}-8$ and $\mathrm{C}-130$ ozone intercomparison on 19 March 2006.

agreement in the relatively small scale changes that occur when $\mathrm{O}_{3}$ remains consistently low (at low altitude in the marine boundary layer) and also at higher altitudes and higher $\mathrm{O}_{3}$ levels (polluted Mexico City airmass). The timeseries for water displays a similar behavior. The large-scale features in the timeseries are well matched while there is less agreement in the finer features at both high (clean free troposphere) and low altitudes (marine boundary layer). The correlation plots (Figs. 2b and 3b) with associated regressions and coefficients of correlation $\left(R^{2}\right)$ offer an additional method for evaluating the likelihood that the instruments sampled the same airmass. $R^{2}$ is defined as

$R^{2}=\frac{[\Sigma(x-\bar{x})(y-\bar{y})]^{2}}{\Sigma(x-\bar{x})^{2} \Sigma(y-\bar{y})^{2}}$

where $\bar{x}$ is the average of the $x$ values and $\bar{y}$ is the average of the $y$ values. Both ozone and water show that the measurements are strongly correlated as evident by the high $R^{2}$ value. Although it is not easy to discern in the time series for
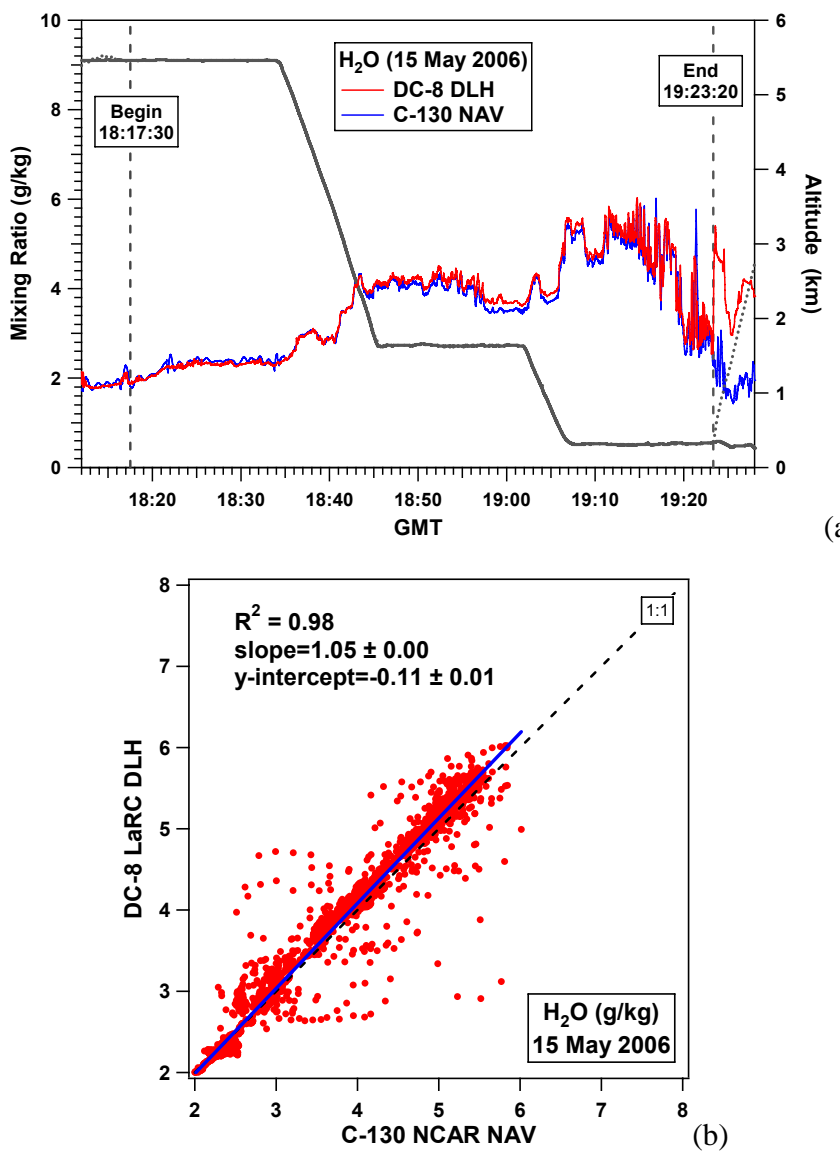

Fig. 3. (a) Timeseries for water during the intercomparison portion of the 15 May 2006 flight. The dotted line indicates the DC- 8 altitude, solid thick line the C-130 altitude, red line DC- 8 ozone, and blue line C-130 ozone. (b) Scatter plot and orthogonal distance regression for the DC- 8 and C-130 water intercomparison on 15 May 2006.

water, there is a slight time lag in one of the water measurements. This is evident in Fig. 3b where data points depart the tighter cluster in curved lines. In general the spread in the data appears larger for water than ozone, however, this may be due in part to the smaller range in the $x$ and $y$ scales for water. The high $R^{2}$ value for both ozone and water nevertheless indicate that the two aircraft are most likely sampling the same airmass.

Intercomparison analysis was conducted during each stage of data submission: (1) comparison of field data (blind), (2) comparison of preliminary data (not blind), and (3) comparison of final data (not blind). These analyses and the distribution of results were carried out by the Measurement Comparison Working Group (MCWG). The primary responsibility of the MCWG included providing for secure field data submission to facilitate the "blind" comparison, analyzing data for each stage of data submission, and disseminating the results within the science team and to the 
Table 2. Summary of Intercomparison Measurements.

\begin{tabular}{|c|c|c|c|c|c|}
\hline Species & Technique $^{\mathrm{a}}$ & Aircraft & Principal Investigator & Uncertainty $^{\mathrm{b}}$ & $\begin{array}{l}\text { Confidence } \\
\text { Level }\end{array}$ \\
\hline \multirow[t]{2}{*}{$\mathrm{CO}$} & UVF & C-130 & T. Campos, NCAR & $10 \%$ & Contact PI \\
\hline & DACOM & DC-8 & G. Sachse, NASA LaRC & $2 \%$ or 2 ppbv ( 1 sigma $p=1 \%$ or 1 ppbv, $a=2 \%$ ) & Contact PI \\
\hline \multirow[t]{3}{*}{$\mathrm{H}_{2} \mathrm{O}$} & Cryo & DC-8 & J. Barrick, NASA LaRC, UND/NSERC & $5 \%$ & 2 sigma \\
\hline & Cryo & C-130 & Allen Schanot, NCAR/RAF & $\pm 0.5 \mathrm{C} ; \pm 1 \mathrm{C}$ below a dp of $-60 \mathrm{C}$ & Contact PI \\
\hline & DLH & DC-8 & G. Diskin, NASA LaRC & $5 \%(1$ sigma $p=1 \%$ or 0.05 ppmv, $a=5 \%$ or $1 \mathrm{ppmv})$ & Contact PI \\
\hline \multirow[t]{2}{*}{ NO } & CLD & C-130 & A. Weinheimer, NCAR & $10 \mathrm{pptv}$ or $10 \%$ & 1 sigma \\
\hline & CLD & DC-8 & G. Huey, GIT & $(6.83,85.71) 25 \%^{\mathrm{c}}$ & 2 sigma \\
\hline \multirow[t]{2}{*}{$\mathrm{NO}_{2}$} & CLD & $\mathrm{C}-130$ & A. Weinheimer, NCAR & 20 pptv or $15 \%$ & 1 sigma \\
\hline & TD-LIF & DC-8 & R. Cohen, UC Berkeley & $15 \mathrm{pptv}+(0.05 \times \text { value })^{\mathrm{d}}(a=5 \%)$ & 2 sigma \\
\hline \multirow{2}{*}{$\mathrm{O}_{3}$} & CLD & DC-8 & M. Avery, NASA LaRC & 3 ppb or $3 \%$ dry air, $5-7 \%$ moist air $(p<1$ ppbv, 2 sigma $)$ & Contact PI \\
\hline & CLD & C-130 & A. Weinheimer, NCAR & $0.1 \mathrm{ppbv}$ or $5 \%$ & 1 sigma \\
\hline \multirow{3}{*}{$\mathrm{SO}_{2}$} & CIMS & DC-8 & G. Huey, GIT & $15 \%$ & 2 sigma \\
\hline & UVF & C-130 & J. Holloway, NOAA & $15 \%+0.8 \mathrm{ppbv}(p=0.8 \mathrm{ppbv}, 2$ sigma $)$ & 2 sigma \\
\hline & CIMS & $\mathrm{C}-130$ & P. Wennberg, CIT & $35 \%+0.2$ ppbv $+0.2 \times$ formic acid & Contact PI \\
\hline \multirow[t]{2}{*}{$\mathrm{HCN}$} & CIMS & C-130 & P. Wennberg, CIT & $\pm 20 \%+50 \mathrm{pptv}$ & Contact PI \\
\hline & PANAK & DC-8 & H. Singh, NASA ARC & $\pm 15 \%$ & 1 sigma \\
\hline \multirow[t]{3}{*}{$\mathrm{CH}_{3} \mathrm{CN}$} & TOGA & C-130 & E. Apel, NCAR & $20 \%$ & 1 sigma \\
\hline & PANAK & DC-8 & H. Singh, NASA ARC & $\pm 15 \%$ & 1 sigma \\
\hline & PTRMS & $\mathrm{C}-130$ & T. Karl, NCAR/ACD & $35 \%(a=15 \%)$ & 1 sigma \\
\hline \multirow[t]{2}{*}{ Propanal } & TOGA & C-130 & E. Apel, NCAR & $20 \%$ & 1 sigma \\
\hline & PANAK & DC-8 & H. Singh, NASA ARC & $50 \%$ & 1 sigma \\
\hline \multirow[t]{3}{*}{$\mathrm{CH}_{2} \mathrm{O}$} & DFG & C-130 & P. Weibring, NCAR & $(13.45,97.67) 17.16 \%^{\mathrm{c}}$ & 2 sigma \\
\hline & TDL & DC-8 & A. Fried, NCAR & $(15.15,269.8) 37.3 \%^{c}$ & 2 sigma \\
\hline & EFD & DC-8 & B. Heikes, URI & $(17.61,81.48) 19.3 \%^{\mathrm{c}}$ & Contact PI \\
\hline \multirow[t]{2}{*}{$\mathrm{CH}_{3} \mathrm{OOH}$} & CIMS & C-130 & P. Wennberg, CIT & $50 \%+250 \mathrm{pptv}$ & Contact PI \\
\hline & EFD & DC-8 & B. Heikes, URI & $135+(0.25 \times$ value $)$ & Contact PI \\
\hline \multirow[t]{3}{*}{$\mathrm{H}_{2} \mathrm{O}_{2}$} & CIMS & C-130 & P. Wennberg, CIT & $25 \%+100 \mathrm{pptv}$ & Contact PI \\
\hline & EFD & DC-8 & B. Heikes, URI & $15+(0.15 \times$ value $)$ & Contact PI \\
\hline & ACCD & DC-8 & D. O'Sullivan, USNA & $30 \mathrm{ppt}+0.35 \times$ value & 2 sigma \\
\hline \multirow[t]{3}{*}{$\mathrm{HNO}_{3}$} & CIMS & C-130 & P. Wennberg, CIT & $30 \%+50 \mathrm{pptv}$ & Contact PI \\
\hline & TD-LIF & DC-8 & R. Cohen, UC Berkeley & $(23.43,97.85) 43.7 \%^{\mathrm{c}}$ & 2 sigma \\
\hline & $\mathrm{MC}$ & DC-8 & R. Talbot, UNH & $<25 \mathrm{pptv}=30-35 \% ; 25-100 \mathrm{pptv}=20 \% ;>100 \mathrm{pptv}=15 \%$ & 1 sigma \\
\hline \multirow[t]{2}{*}{ PAN } & CIGAR & C-130 & F. Flocke, NCAR/ACD & $(p=9 \%, a=10 \%+18 \mathrm{pptv})$ & 2 sigma \\
\hline & PANAK & DC-8 & H. Singh, NASA ARC & $10 \%(>100 \mathrm{pptv}) ; 15 \%(<100 \mathrm{pptv})$ & 1 sigma \\
\hline \multirow[t]{2}{*}{ Total PANs ${ }^{\mathrm{e}}$} & CIGAR & C-130 & F. Flocke, NCAR/ACD & $(p=9 \%, a=10 \%+18 \mathrm{pptv})$ & 2 sigma \\
\hline & TD-LIF & DC-8 & R. Cohen, UC Berkeley & $20 \mathrm{pptv}+(0.1 \times \text { value })^{\mathrm{d}}$ & 2 sigma \\
\hline \multirow[t]{2}{*}{$\mathrm{NO}_{\mathrm{y}}-\mathrm{NO}$} & CLD & $\mathrm{C}-130$ & A. Weinheimer, NCAR & Derived quantity from $\mathrm{NO}_{\mathrm{y}}$ and $\mathrm{NO}$ & Contact PI \\
\hline & TD-LIF & DC-8 & R. Cohen, UC Berkeley & $(13,78) 35 \%^{\mathrm{c}}$ & 2 sigma \\
\hline $\mathrm{OH}$ & CIMS & C-130 & L. Mauldin, NCAR & $35 \%$ & 2 sigma \\
\hline & ATHOS & DC-8 & W. Brune, Penn State & $(a=32 \%, 2$ sigma $)$ & Contact PI \\
\hline $\mathrm{HO}_{2}$ & CIMS & C-130 & C. Cantrell, NCAR & $35 \%$ & 2 sigma \\
\hline & ATHOS & DC-8 & W. Brune, Penn State & $(a=32 \%, 2$ sigma $)$ & Contact PI \\
\hline Acetaldehyde & TOGA & C-130 & E. Apel, NCAR & $20 \%$ & 1 sigma \\
\hline & PANAK & DC-8 & H. Singh, NASA ARC & $50 \%$ & 1 sigma \\
\hline & PTRMS & C-130 & T. Karl, NCAR/ACD & $35 \%$ & 1 sigma \\
\hline Acetone & TOGA & $\mathrm{C}-130$ & E. Apel, NCAR & $20 \%$ & 1 sigma \\
\hline & PANAK & DC-8 & H. Singh, NASA ARC & $20 \%$ & 1 sigma \\
\hline Ethanol & TOGA & C-130 & E. Apel, NCAR & $20 \%$ & 1 sigma \\
\hline & PANAK & DC-8 & H. Singh, NASA ARC & $20 \%$ & 1 sigma \\
\hline MEK & TOGA & C-130 & E. Apel, NCAR & $20 \%$ & 1 sigma \\
\hline & PANAK & DC-8 & H. Singh, NASA ARC & $20 \%$ & 1 sigma \\
\hline & PTRMS & C-130 & T. Karl, NCAR/ACD & $35 \%$ & 1 sigma \\
\hline Methanol & TOGA & C-130 & E. Apel, NCAR & $20 \%$ & 1 sigma \\
\hline & PANAK & DC-8 & H. Singh, NASA ARC & $20 \%$ & 1 sigma \\
\hline & PTRMS & C-130 & T. Karl, NCAR/ACD & $35 \%$ & 1 sigma \\
\hline All NMHCs & WAS & DC-8/C-130 & D. Blake, UCI & $5 \%$ & 1 sigma \\
\hline$j\left(\mathrm{O}_{3}\right)$ & SAFS & DC-8/C-130 & R. Shetter, ARIM/NCAR & See footnote e & Contact PI \\
\hline$j\left(\mathrm{NO}_{2}\right)$ & SAFS & DC-8/C-130 & R. Shetter, ARIM/NCAR & See footnote e & Contact PI \\
\hline & Filt. Rad & DC- 8 & J. Barrick, NASA LaRC & $8 \%$ & 2 sigma \\
\hline
\end{tabular}


Table 2. Continued.

\begin{tabular}{|c|c|c|c|c|c|}
\hline Species & Technique $^{\mathrm{a}}$ & Aircraft & Principal Investigator & Uncertainty $^{\mathrm{b}}$ & $\begin{array}{l}\text { Confidence } \\
\text { Level }\end{array}$ \\
\hline \multirow[t]{2}{*}{$N>3 \mathrm{~nm}$} & $\mathrm{CPC}$ & DC-8 & B. Anderson, LaRC/A. Clarke, U Hawaii & $10 \%$ & Contact PI \\
\hline & $\mathrm{CPC}$ & C-130 & A. Clarke, U Hawaii & $10 \%$ & Contact PI \\
\hline \multirow[t]{2}{*}{$N>10 \mathrm{~nm}(05 / 15)$} & $\mathrm{CPC}$ & DC-8 & B. Anderson, LaRC/A. Clarke, U Hawaii & $5 \%$ & Contact PI \\
\hline & $\mathrm{CPC}$ & C-130 & A. Clarke, U Hawaii & $5 \%$ & Contact PI \\
\hline \multirow[t]{2}{*}{$N>10 \mathrm{~nm}(04 / 17)$} & $\mathrm{CPC}$ & DC-8 & B. Anderson, LaRC/A. Clarke, U Hawaii & $5 \%$ & Contact PI \\
\hline & $\mathrm{CPC}$ & $\mathrm{C}-130$ & A. Clarke, U Hawaii & $5 \%$ & Contact PI \\
\hline \multirow[t]{2}{*}{ Hot CN (03/19) } & $\mathrm{CPC}$ & DC-8 & B. Anderson, LaRC/A. Clarke, U Hawaii & $5 \%$ & Contact PI \\
\hline & $\mathrm{CPC}$ & $\mathrm{C}-130$ & A. Clarke, U Hawaii & $5 \%$ & Contact PI \\
\hline \multirow[t]{2}{*}{ Hot CN $(05 / 15)$} & $\mathrm{CPC}$ & DC-8 & B. Anderson, LaRC/A. Clarke, U Hawaii & $5 \%$ & Contact PI \\
\hline & $\mathrm{CPC}$ & $\mathrm{C}-130$ & A. Clarke, U Hawaii & $5 \%$ & Contact PI \\
\hline \multirow[t]{2}{*}{ N_DMA } & DMA & DC-8 & B. Anderson, LaRC/A. Clarke, U Hawaii & $15 \%$ & Contact PI \\
\hline & DMA & $\mathrm{C}-130$ & A. Clarke, U Hawaii & $15 \%$ & Contact PI \\
\hline \multirow[t]{2}{*}{ N_OPC } & OPC & DC-8 & B. Anderson, LaRC/A. Clarke, U Hawaii & $15 \%$ & Contact PI \\
\hline & $\mathrm{OPC}$ & C-130 & A. Clarke, U Hawaii & $15 \%$ & Contact PI \\
\hline \multirow[t]{2}{*}{ N_APS } & APS & DC-8 & B. Anderson, LaRC/A. Clarke, U Hawaii & $15 \%$ & Contact PI \\
\hline & APS & $\mathrm{C}-130$ & A. Clarke, U Hawaii & $15 \%$ & Contact PI \\
\hline \multirow[t]{2}{*}{ Nsub } & OPC & DC-8 & B. Anderson, LaRC/A. Clarke, U Hawaii & $15 \%$ & Contact PI \\
\hline & $\mathrm{OPC}$ & $\mathrm{C}-130$ & A. Clarke, U Hawaii & $15 \%$ & Contact PI \\
\hline \multirow[t]{2}{*}{ Nsuper } & OPC & DC-8 & B. Anderson, LaRC/A. Clarke, U Hawaii & $15 \%$ & Contact PI \\
\hline & $\mathrm{OPC}$ & C-130 & A. Clarke, U Hawaii & $15 \%$ & Contact PI \\
\hline \multirow[t]{2}{*}{ N_150C_DMA } & DMA & DC-8 & B. Anderson, LaRC/A. Clarke, U Hawaii & $15 \%$ & Contact PI \\
\hline & DMA & $\mathrm{C}-130$ & A. Clarke, U Hawaii & $15 \%$ & Contact PI \\
\hline \multirow[t]{2}{*}{ N_150C_OPC } & OPC & DC-8 & B. Anderson, LaRC/A. Clarke, U Hawaii & $15 \%$ & Contact PI \\
\hline & $\mathrm{OPC}$ & $\mathrm{C}-130$ & A. Clarke, U Hawaii & $15 \%$ & Contact PI \\
\hline \multirow[t]{2}{*}{ Nsub_150C } & OPC & DC-8 & B. Anderson, LaRC/A. Clarke, U Hawaii & $15 \%$ & Contact PI \\
\hline & $\mathrm{OPC}$ & C-130 & A. Clarke, U Hawaii & $15 \%$ & Contact PI \\
\hline \multirow[t]{2}{*}{ Nsuper_150C } & OPC & DC-8 & B. Anderson, LaRC/A. Clarke, U Hawaii & $15 \%$ & Contact PI \\
\hline & $\mathrm{OPC}$ & C-130 & A. Clarke, U Hawaii & $15 \%$ & Contact PI \\
\hline \multirow[t]{2}{*}{ N_300C_DMA } & DMA & DC-8 & B. Anderson, LaRC/A. Clarke, U Hawaii & $15 \%$ & Contact PI \\
\hline & DMA & $\mathrm{C}-130$ & A. Clarke, U Hawaii & $15 \%$ & Contact PI \\
\hline \multirow[t]{2}{*}{ N_300C_OPC } & OPC & DC-8 & B. Anderson, LaRC/A. Clarke, U Hawaii & $15 \%$ & Contact PI \\
\hline & OPC & C-130 & A. Clarke, U Hawaii & $15 \%$ & Contact PI \\
\hline \multirow[t]{2}{*}{ Nsub_300C } & OPC & DC-8 & B. Anderson, LaRC/A. Clarke, U Hawaii & $15 \%$ & Contact PI \\
\hline & $\mathrm{OPC}$ & C-130 & A. Clarke, U Hawaii & $15 \%$ & Contact PI \\
\hline Nsuper_300C & OPC & DC-8 & B. Anderson, LaRC/A. Clarke, U Hawaii & $15 \%$ & Contact PI \\
\hline & $\mathrm{OPC}$ & $\mathrm{C}-130$ & A. Clarke, U Hawaii & $15 \%$ & Contact PI \\
\hline N_400C_OPC & OPC & DC-8 & B. Anderson, LaRC/A. Clarke, U Hawaii & $15 \%$ & Contact PI \\
\hline & OPC & C-130 & A. Clarke, U Hawaii & $15 \%$ & Contact PI \\
\hline Nsub_400C & OPC & DC-8 & B. Anderson, LaRC/A. Clarke, U Hawaii & $15 \%$ & Contact PI \\
\hline & $\mathrm{OPC}$ & C-130 & A. Clarke, U Hawaii & $15 \%$ & Contact PI \\
\hline Nsuper_400C & OPC & DC-8 & B. Anderson, LaRC/A. Clarke, U Hawaii & $15 \%$ & Contact PI \\
\hline & $\mathrm{OPC}$ & $\mathrm{C}-130$ & A. Clarke, U Hawaii & $15 \%$ & Contact PI \\
\hline V_DMA & DMA & DC-8 & B. Anderson, LaRC/A. Clarke, U Hawaii & $30 \%$ & Contact PI \\
\hline & DMA & C-130 & A. Clarke, U Hawaii & $30 \%$ & Contact PI \\
\hline V_OPC & OPC & DC-8 & B. Anderson, LaRC/A. Clarke, U Hawaii & $30 \%$ & Contact PI \\
\hline & $\mathrm{OPC}$ & C-130 & A. Clarke, U Hawaii & $30 \%$ & Contact PI \\
\hline V_APS & APS & DC-8 & B. Anderson, LaRC/A. Clarke, U Hawaii & $30 \%$ & Contact PI \\
\hline & APS & C-130 & A. Clarke, U Hawaii & $30 \%$ & Contact PI \\
\hline Vsub & OPC & DC-8 & B. Anderson, LaRC/A. Clarke, U Hawaii & $30 \%$ & Contact PI \\
\hline & OPC & C-130 & A. Clarke, U Hawaii & $30 \%$ & Contact PI \\
\hline Vsuper & OPC & DC-8 & B. Anderson, LaRC/A. Clarke, U Hawaii & $30 \%$ & Contact PI \\
\hline & $\mathrm{OPC}$ & C-130 & A. Clarke, U Hawaii & $30 \%$ & Contact PI \\
\hline V_150C_DMA & DMA & DC-8 & B. Anderson, LaRC/A. Clarke, U Hawaii & $30 \%$ & Contact PI \\
\hline & DMA & C-130 & A. Clarke, U Hawaii & $30 \%$ & Contact PI \\
\hline V_150C_OPC & OPC & DC-8 & B. Anderson, LaRC/A. Clarke, U Hawaii & $30 \%$ & Contact PI \\
\hline & $\mathrm{OPC}$ & C-130 & A. Clarke, U Hawaii & $30 \%$ & Contact PI \\
\hline Vsub_150C & OPC & DC-8 & B. Anderson, LaRC/A. Clarke, U Hawaii & $30 \%$ & Contact PI \\
\hline & $\mathrm{OPC}$ & C-130 & A. Clarke, U Hawaii & $30 \%$ & Contact PI \\
\hline
\end{tabular}


Table 2. Continued.

\begin{tabular}{|c|c|c|c|c|c|}
\hline Species & Technique $^{\mathrm{a}}$ & Aircraft & Principal Investigator & Uncertainty $^{\mathrm{b}}$ & $\begin{array}{l}\text { Confidence } \\
\text { Level }\end{array}$ \\
\hline \multirow[t]{2}{*}{ Vsuper_150C } & $\mathrm{OPC}$ & DC-8 & B. Anderson, LaRC/A. Clarke, U Hawaii & $30 \%$ & Contact PI \\
\hline & OPC & C-130 & A. Clarke, U Hawaii & $30 \%$ & Contact PI \\
\hline \multirow[t]{2}{*}{ V_300C_DMA } & DMA & DC-8 & B. Anderson, LaRC/A. Clarke, U Hawaii & $30 \%$ & Contact PI \\
\hline & DMA & C-130 & A. Clarke, U Hawaii & $30 \%$ & Contact PI \\
\hline \multirow[t]{2}{*}{ V_300C_OPC } & OPC & DC-8 & B. Anderson, LaRC/A. Clarke, U Hawaii & $30 \%$ & Contact PI \\
\hline & $\mathrm{OPC}$ & C-130 & A. Clarke, U Hawaii & $30 \%$ & Contact PI \\
\hline \multirow[t]{2}{*}{ Vsub_300C } & OPC & DC-8 & B. Anderson, LaRC/A. Clarke, U Hawaii & $30 \%$ & Contact PI \\
\hline & $\mathrm{OPC}$ & C-130 & A. Clarke, U Hawaii & $30 \%$ & Contact PI \\
\hline \multirow[t]{2}{*}{ Vsuper_300C } & OPC & DC-8 & B. Anderson, LaRC/A. Clarke, U Hawaii & $30 \%$ & Contact PI \\
\hline & OPC & C-130 & A. Clarke, U Hawaii & $30 \%$ & Contact PI \\
\hline \multirow[t]{2}{*}{ V_400C_OPC } & OPC & DC-8 & B. Anderson, LaRC/A. Clarke, U Hawaii & $30 \%$ & Contact PI \\
\hline & $\mathrm{OPC}$ & C-130 & A. Clarke, U Hawaii & $30 \%$ & Contact PI \\
\hline \multirow[t]{2}{*}{ Vsub_400C } & OPC & DC-8 & B. Anderson, LaRC/A. Clarke, U Hawaii & $30 \%$ & Contact PI \\
\hline & $\mathrm{OPC}$ & C-130 & A. Clarke, U Hawaii & $30 \%$ & Contact PI \\
\hline \multirow[t]{2}{*}{ Vsuper_400C } & OPC & DC-8 & B. Anderson, LaRC/A. Clarke, U Hawaii & $30 \%$ & Contact PI \\
\hline & OPC & $\mathrm{C}-130$ & A. Clarke, U Hawaii & $30 \%$ & Contact PI \\
\hline \multirow[t]{3}{*}{$\mathrm{SO}_{4}^{=}$} & MC & DC-8 & J. Dibb, UNH & $10 \%+5 \mathrm{pptv}$ & 1 sigma \\
\hline & AMS & C-130 & J. Jimenez, U CO & $0.04 \mu \mathrm{g} \mathrm{s} \mathrm{m}^{-3}(a=35 \%, 2 \text { sigma })^{\mathrm{g}}$ & 2 sigma \\
\hline & PILS & $\mathrm{C}-130$ & R. Weber, GIT & Conc $>2 \times \mathrm{LOD}=20 \%$ Conc $<=2 \times \mathrm{LOD}=40 \%$ & 1 sigma \\
\hline \multirow{2}{*}{$\mathrm{NO}_{3}^{-}$} & AMS & C-130 & J. Jimenez, U CO & $0.06 \mu \mathrm{g} \mathrm{s} \mathrm{m}^{-3}(a=35 \%, 2 \text { sigma })^{\mathrm{g}}$ & 2 sigma \\
\hline & PILS & C-130 & R. Weber, GIT & Conc $>2 \times \mathrm{LOD}=20 \%$ Conc $<=2 \times \mathrm{LOD}=40 \%$ & 1 sigma \\
\hline \multirow[t]{2}{*}{$\mathrm{NH}_{4}^{+}$} & AMS & C-130 & J. Jimenez, U CO & $0.36 \mu \mathrm{g} \mathrm{s} \mathrm{m}^{-3}(a=35 \%, 2 \text { sigma })^{\mathrm{g}}$ & 2 sigma \\
\hline & PILS & C-130 & R. Weber, GIT & Conc $>2 \times \mathrm{LOD}=20 \%$ Conc $<=2 \times \mathrm{LOD}=40 \%$ & 1 sigma \\
\hline \multirow[t]{2}{*}{ Scatt $450 \mathrm{~nm}$} & TSI Nephelometer & DC-8 & B. Anderson, LaRC/A. Clarke, U Hawaii & $10 \%$ or $0.5 \mathrm{Mm}^{-1}$ & Contact PI \\
\hline & TSI Nephelometer & C-130 & A. Clarke, U Hawaii & $10 \%$ or $0.5 \mathrm{Mm}^{-1}$ & Contact PI \\
\hline \multirow[t]{2}{*}{ Scatt $550 \mathrm{~nm}$} & TSI Nephelometer & DC-8 & B. Anderson, LaRC/A. Clarke, U Hawaii & $10 \%$ or $0.5 \mathrm{Mm}^{-1}$ & Contact PI \\
\hline & TSI Nephelometer & C-130 & A. Clarke, U Hawaii & $10 \%$ or $0.5 \mathrm{Mm}^{-1}$ & Contact PI \\
\hline \multirow[t]{2}{*}{ Scatt $700 \mathrm{~nm}$} & TSI Nephelometer & DC-8 & B. Anderson, LaRC/A. Clarke, U Hawaii & $10 \%$ or $0.5 \mathrm{Mm}^{-1}$ & Contact PI \\
\hline & TSI Nephelometer & C-130 & A. Clarke, U Hawaii & $10 \%$ or $0.5 \mathrm{Mm}^{-1}$ & Contact PI \\
\hline \multirow[t]{2}{*}{ Scattsub $550 \mathrm{~nm}$} & RR Nephelometer & DC-8 & B. Anderson, LaRC/A. Clarke, U Hawaii & $10 \%$ or $0.5 \mathrm{Mm}^{-1}$ & Contact PI \\
\hline & RR Nephelometer & C-130 & A. Clarke, U Hawaii & $10 \%$ or $0.5 \mathrm{Mm}^{-1}$ & Contact PI \\
\hline \multirow[t]{2}{*}{ Abs $470 \mathrm{~nm}$} & PSAP & DC-8 & B. Anderson, LaRC/A. Clarke, U Hawaii & $20 \%$ or $0.5 \mathrm{Mm}^{-1}$ & Contact PI \\
\hline & PSAP & C-130 & A. Clarke, U Hawaii & $20 \%$ or $0.5 \mathrm{Mm}^{-1}$ & Contact PI \\
\hline \multirow[t]{2}{*}{ Abs $530 \mathrm{~nm}$} & PSAP & DC-8 & B. Anderson, LaRC/A. Clarke, U Hawaii & $20 \%$ or $0.5 \mathrm{Mm}^{-1}$ & Contact PI \\
\hline & PSAP & C-130 & A. Clarke, U Hawaii & $20 \%$ or $0.5 \mathrm{Mm}^{-1}$ & Contact PI \\
\hline \multirow[t]{2}{*}{ Abs $660 \mathrm{~nm}$} & PSAP & DC-8 & B. Anderson, LaRC/A. Clarke, U Hawaii & $20 \%$ or $0.5 \mathrm{Mm}^{-1}$ & Contact PI \\
\hline & PSAP & C-130 & A. Clarke, U Hawaii & $20 \%$ or $0.5 \mathrm{Mm}^{-1}$ & Contact PI \\
\hline
\end{tabular}

a For an explanation of "Technique", the reader is referred to the individual PI files located on the INTEX-B website (http://www-air.larc.nasa.gov/missions/intex-b/intexb.html) under the Current Archive Status link.

$\mathrm{b}$ Total uncertainty unless otherwise specified. Precision $(p)$ and accuracy $(a)$ given in parentheses.

${ }^{c}$ Absolute uncertainty reported point-by-point. Percent uncertainty for the intercomparison period is calculated, minimum and maximum given in parentheses, median given outside the parentheses.

${ }^{\mathrm{d}}$ Uncertainty for one second data reported point-by-point in file header. For consistency, values shown are PI estimates for $60 \mathrm{~s}$ averages.

e No PI reported uncertainty.

${ }^{\mathrm{f}}$ PANs = Peroxy alkyl nitrates, formula R-C(O)OONO2, with $R=$ liphatic, olefinic, or substituted aliphatic or olefinic substituent.

$\mathrm{g}$ Uncertainty given for 12 second integration time. For further details, PI refers the reader to Dunlea et al. (2009) and Bahreini et al. (2009).

atmospheric community at large. In stage one, the blind comparison of field data, PIs submitted data within $24 \mathrm{~h}$ to a few days after the flight to an ftp site which was "blind" to the science team for a period of time until both paired comparison data were submitted. For example, the $\mathrm{CO}$ data was not available to the science team until both NSF C-130 and NASA DC-8 PIs submitted their CO data for the intercomparison flight. The MCWG then assessed the consistency between the paired DC- 8 and C-130 measurements/instruments and released the comparison results and the data to the science team. In the preliminary data stage, data were compared again after allowing the PIs to apply post mission calibration and additional processing/correction procedures to their data. The MCWG presented these 
Table 3a. Statistical results of DC-8/C-130 intercomparison. Photochemical precursors and gas phase tracers. NOTE: Technique is listed as X (C-130) vs. Y (DC-8).

\begin{tabular}{|c|c|c|c|c|c|c|c|c|c|c|c|}
\hline \multirow[t]{2}{*}{ Species } & \multirow[t]{2}{*}{ Technique } & \multirow[t]{2}{*}{ Units } & \multirow[t]{2}{*}{ Slope } & \multirow[t]{2}{*}{ Intercept } & \multirow[t]{2}{*}{$R^{2}$} & \multicolumn{3}{|c|}{ Ratio Percentiles } & \multirow[t]{2}{*}{ \# Pts } & \multicolumn{2}{|c|}{ Range } \\
\hline & & & & & & 25 th & 50th & 75th & & Min & Max \\
\hline $\mathrm{CO}$ & UVF vs. DACOM & ppbv & $1.09 \pm 0.00$ & $-5.1 \pm 0.2$ & 0.99 & & & & 7823 & 68.5 & 223 \\
\hline \multirow{2}{*}{$\mathrm{H}_{2} \mathrm{O}$} & Cryo vs. DLH & $\mathrm{g} \mathrm{kg}^{-1}$ & $0.92 \pm 0.00$ & $0.15 \pm 0.0$ & 0.99 & & & & 8928 & $>.0006$ & 16.5 \\
\hline & Cryo vs. Cryo & $\mathrm{g} \mathrm{kg}^{-1}$ & $0.94 \pm 0.00$ & $0.05 \pm 0.0$ & 0.99 & & & & 9050 & 0.02 & 16.5 \\
\hline NO & CLD vs. CLD & pptv & $0.95 \pm 0.01$ & $13.1 \pm 0.2$ & 0.81 & & & & 5277 & LOD & 205 \\
\hline $\mathrm{NO}_{2}$ & CLD vs. TD-LIF & pptv & $1.20 \pm 0.01$ & $-39 \pm 1$ & 0.87 & & & & 2254 & LOD & 796 \\
\hline $\mathrm{O}_{3}$ & CLD vs. CLD & ppbv & $1.00 \pm 0.00$ & $-1.0 \pm 0.1$ & 0.99 & & & & 6408 & 26.2 & 133 \\
\hline \multirow[t]{2}{*}{$\mathrm{SO}_{2}$} & CIMS vs. CIMS & pptv & $0.56 \pm 0.00$ & $3 \pm 16$ & 0.98 & & & & 307 & 3 & 21610 \\
\hline & UVF vs. CIMS & pptv & $0.86 \pm 0.01$ & $-486 \pm 27$ & 0.97 & & & & 434 & 230 & 14700 \\
\hline $\mathrm{HCN}$ & CIMS vs. PANAK & pptv & & & 0.37 & 0.50 & 0.69 & 0.90 & 22 & 150 & 2272 \\
\hline \multirow{2}{*}{$\mathrm{CH}_{3} \mathrm{CN}^{\mathrm{a}}$} & TOGA vs. PANAK & pptv & & & 0.06 & 0.78 & 1.02 & 1.15 & 16 & 0.03 & 0.29 \\
\hline & PTRMS vs. PANAK & pptv & & & 0.61 & 0.64 & 0.83 & 0.95 & 16 & 0.04 & 0.29 \\
\hline Propanal $^{\mathrm{a}}$ & TOGA vs. PANAK & pptv & & & 0.38 & 0.63 & 1.23 & 1.86 & 10 & 0.005 & 0.18 \\
\hline
\end{tabular}

a Online files found in VOCs link at http://www-air.larc.nasa.gov/missions/intex-b/intexb.html under the Measurement Comparisons: MILAGRO/INTEX-B/IMPEX link.

Table 3b. Statistical results of DC-8/C-130 intercomparison. Photochemical products. NOTE: Technique is listed as X (C-130) vs. Y (DC-8).

\begin{tabular}{|c|c|c|c|c|c|c|c|c|c|c|c|}
\hline \multirow[t]{2}{*}{ Species } & \multirow[t]{2}{*}{ Technique } & \multirow[t]{2}{*}{ Units } & \multirow[t]{2}{*}{ Slope } & \multirow[t]{2}{*}{ Intercept } & \multirow[t]{2}{*}{$R^{2}$} & \multicolumn{3}{|c|}{ Ratio Percentiles } & \multirow[t]{2}{*}{ \# Pts } & \multicolumn{2}{|c|}{ Range } \\
\hline & & & & & & 25 th & 50th & 75th & & Min & Max \\
\hline \multirow{2}{*}{$\mathrm{CH}_{2} \mathrm{O}$} & DFG vs. EFD & pptv & $1.12 \pm 0.09$ & $-401 \pm 152$ & 0.88 & & & & 24 & LOD & 3687 \\
\hline & DFG vs. TDL & pptv & $1.01 \pm 0.03$ & $19 \pm 33$ & 0.95 & & & & 67 & LOD & 3861 \\
\hline $\mathrm{CH}_{3} \mathrm{OOH}$ & CIMS vs. EFD & pptv & & & 0.30 & 0.87 & 1.13 & 1.41 & 26 & 217 & 2286 \\
\hline \multirow[t]{2}{*}{$\mathrm{H}_{2} \mathrm{O}_{2}$} & CIMS vs. EFD & pptv & $1.24 \pm 0.04$ & $-19 \pm 67$ & 0.92 & & & & 74 & 41 & 2809 \\
\hline & CIMS vs. ACCD & pptv & $0.84 \pm 0.02$ & $313 \pm 21$ & 0.83 & & & & 392 & 80 & 2314 \\
\hline \multirow[t]{2}{*}{$\mathrm{HNO}_{3}$} & CIMS vs. MC & pptv & $1.21 \pm 0.04$ & $-3 \pm 14$ & 0.88 & & & & 98 & 10 & 1302 \\
\hline & CIMS vs. TDLIF & pptv & & & 0.63 & 0.57 & 0.66 & 0.80 & 45 & 78 & 1749 \\
\hline PAN & CIGAR vs. PANAK & pptv & $1.68 \pm 0.16$ & $-185 \pm 59$ & 0.77 & & & & 33 & 2 & 1986 \\
\hline Total PAN & CIGAR vs. TDLIF & pptv & $1.35 \pm 0.03$ & $-83 \pm 10$ & 0.94 & & & & 157 & LOD & 2175 \\
\hline $\mathrm{NO}_{\mathrm{y}}-\mathrm{NO}$ & CLD vs. TD-LIF & pptv & $0.92 \pm 0.01$ & $51 \pm 18$ & 0.97 & & & & 143 & 133 & 5559 \\
\hline
\end{tabular}

results to the science team at the post-mission data workshop. In the comparison of final data (not blind), PIs submitted final data with uncertainty estimates. These results are archived online (http://www-air.larc.nasa.gov/missions/ intex-b/intexb-meas-comparison.htm) and summarized here.

In addition to the inter-platform comparisons, intraplatform comparisons were made whenever possible. Since both instruments were located on the same aircraft, these comparisons were not limited to the three intercomparison periods discussed previously, rather they could span the entire mission.

As previously stated, the primary goal of this paper is to present a comprehensive overview of the INTEXB/MILAGRO/IMPEX intercomparison results and provide a record of the measurement consistency. The level of the agreement between the measurements may depend on a number of factors, including calibration, instrument time re- sponse, and measurement techniques. For the comparison of the aerosol measurements, the particle size range of the measurements should be a critical consideration. The information summarized in Table 2 and Tables 3-5 is critical to determine if observations made from different platforms/instruments are consistent within the PI reported uncertainties. This is necessary when deciding if multiple data sets should be used in integrated analysis. At the same time, users are cautioned that differences between measurements can still be significant, even though they are technically consistent within the combined uncertainties quoted by the PIs. In addition, this overview paper does not attempt to describe the complexities of the various measurement techniques. Any interpretation of the results of these studies should be done in consultation with the individual instrument PIs (provided in Table 2). 
Table 3c. Statistical results of DC-8/C-130 intercomparison. Photochemical radicals. NOTE: Technique is listed as X (C-130) vs. Y (DC-8).

\begin{tabular}{|c|c|c|c|c|c|c|c|c|c|c|c|}
\hline \multirow[t]{2}{*}{ Species } & \multirow[t]{2}{*}{ Technique } & \multirow[t]{2}{*}{ Units } & \multirow[t]{2}{*}{ Slope } & \multirow[t]{2}{*}{ Intercept } & \multirow[t]{2}{*}{$R^{2}$} & \multicolumn{3}{|c|}{ Ratio Percentiles } & \multirow[t]{2}{*}{ \# Pts } & \multicolumn{2}{|c|}{ Range } \\
\hline & & & & & & 25 th & 50 th & 75 th & & Min & Max \\
\hline $\mathrm{OH}$ & CIMS vs. ATHOS & pptv & & & 0.03 & 0.41 & 0.81 & 1.06 & 266 & 0.003 & 0.62 \\
\hline $\mathrm{HO}_{2}$ & CIMS vs. ATHOS & pptv & & & 0.59 & 0.98 & 1.23 & 1.73 & 107 & LOD & 64.4 \\
\hline
\end{tabular}

Table 3d. Statistical results of DC-8/C-130 intercomparison. Oxygenated volatile organic carbons. NOTE: Technique is listed as X (C-130) vs. Y (DC-8).

\begin{tabular}{|c|c|c|c|c|c|c|c|c|c|c|c|}
\hline \multirow[t]{2}{*}{ Species } & \multirow[t]{2}{*}{ Technique } & \multirow[t]{2}{*}{ Units } & \multirow[t]{2}{*}{ Slope } & \multirow[t]{2}{*}{ Intercept } & \multirow[t]{2}{*}{$R^{2}$} & \multicolumn{3}{|c|}{ Ratio Percentiles } & \multirow[t]{2}{*}{ \# Pts } & \multicolumn{2}{|c|}{ Range } \\
\hline & & & & & & 25 th & 50 th & 75th & & Min & $\operatorname{Max}$ \\
\hline \multirow[t]{2}{*}{ Acetaldehyde $^{\mathrm{a}}$} & TOGA vs. PANAK & pptv & 1.27 & 0.02 & 0.93 & & & & 14 & 0.02 & 1.3 \\
\hline & PTRMS vs. PANAK & pptv & $1.31 \pm 0.21$ & $0.03 \pm 0.10$ & 0.78 & & & & 12 & 0.04 & 1.3 \\
\hline Acetone & TOGA vs. PANAK & pptv & & & 0.50 & 1.05 & 1.42 & 1.82 & 16 & 0.24 & 3.0 \\
\hline Ethanol $^{\mathrm{a}}$ & TOGA vs. PANAK & pptv & & & & & & & 4 & & \\
\hline MEK $^{\mathrm{a}}$ & TOGA vs. PANAK & pptv & $0.62 \pm 0.07$ & $0.00 \pm 0.01$ & 0.84 & & & & 16 & 0.01 & 0.22 \\
\hline \multirow[t]{2}{*}{ Methanol $^{\mathrm{a}}$} & TOGA vs. PANAK & pptv & & & 0.47 & 1.31 & 2.51 & 3.36 & 16 & 0.20 & 6.6 \\
\hline & PTRMS vs. PANAK & pptv & & & 0.25 & 1.60 & 2.09 & 2.57 & 16 & 0.25 & 11.5 \\
\hline
\end{tabular}

${ }^{a}$ Online files found in VOCs link at http://www-air.larc.nasa.gov/missions/intex-b/intexb.html under the Measurement Comparisons: MILAGRO/INTEX-B/IMPEX link.

\section{Data process procedures and statistical assessment}

The quantitative assessment of measurement/instrument consistency was based on statistical analysis of the intercomparison data. This required the merging of data to a common timeline. Merging was easiest when measurements were conducted with the same timing and integration period; however, it is not unusual for instruments based on different techniques to require different integration times to measure the same species/parameter or that instruments on different platforms are not well synchronized. For cases where instruments had the same integration period, but were not synchronized, the data were merged to ensure at least $50 \%$ sampling time overlap. For paired measurements with different integration time intervals, the shorter integration time measurements were merged into the longer time interval when measurements at the shorter time interval overlapped at least $50 \%$ of the longer time interval. These merged data pairs were used to quantitatively assess measurement consistency through linear regression analysis, when applicable, or descriptive statistics based on the ratio (DC-8/C-130) of the paired data points. The linear regression slopes and intercepts can be used to describe the level of the measurement agreement when a high enough level of correlation exists. Here, this criteria has been defined as an $R^{2}$ value of 0.75 . Lower $R^{2}$ values are typically encountered when the range of variation is limited in comparison to the uncertainties of the measurements and/or other instrument issues exist. When $R^{2}$ is below the threshold of 0.75 , the median and percentile values of the DC-8/C-130 ratio have been used to express the level of consistency between the paired data. In addition, the absolute (or arithmetic) difference between paired data may be used in some cases (with combined uncertainties) to gain additional insight.

Statistical comparisons presented here have been based on Orthogonal Distance Regression (ODR). Orthogonal distance regression is a regression technique similar to ordinary least squares (OLS) fit with the stipulation that both $\mathrm{x}$ and $\mathrm{y}$ are independent variables with errors. ODR minimizes sum of the squares of the orthogonal distances rather than the vertical distances (as in OLS). ODR is generally equivalent to

$\min _{\boldsymbol{\beta}, \delta, \varepsilon} \frac{1}{2} \sum_{i=1}^{n}\left(w_{\varepsilon_{i}} \varepsilon_{i}^{2}+w_{\delta_{i}} \delta_{i}^{2}\right)$

subject to $y_{i}+\varepsilon_{i}=f\left(x_{i}+\delta_{i} ; \boldsymbol{\beta}\right)$ where $\varepsilon_{i}$ is the error in $y$, $\delta_{i}$ the error in $x, w_{\varepsilon_{i}}$ and $w_{\delta_{i}}$ weighting factors, and $\boldsymbol{\beta}$ a vector of parameters to be determined (slope and intercept in this case), (Zwolak et al., 2007). Note that a weighted ODR $\left(w_{\varepsilon_{i}}\right.$ and $\left.w_{\delta_{i}} \neq 1\right)$ is necessary when observations $x_{i}$ and $y_{i}$ are heteroscedastic (variance changes with $i$ ), (Boggs et al., 1988). It has been shown that ODR performs at least as well and in many cases significantly better than Ordinary Least Squares (OLS), especially when $d \sigma_{\varepsilon} / \sigma_{\delta}<2$, (Boggs et al., 1988). Boggs et al. (1988) have shown that ODR results in smaller bias, variance, and mean square error (mse) than OLS, except possibly when significant outliers are present in the data. For the bias of the parameter, $\boldsymbol{\beta}$, and function estimates, $f\left(x_{i} ; \boldsymbol{\beta}\right)$, OLS is statistically better only $2 \%$ of the time while ODR is significantly better $50 \%$ of the time. Results for the variance and mse of the parameter and function 
Table 3e. Statistical results of DC-8/C-130 intercomparison. Nonmethane hydrocarbons, halocarbons, alkylnitrates, and organic sulfur compounds. NOTE: Technique is listed as X (C-130) vs. Y (DC-8).

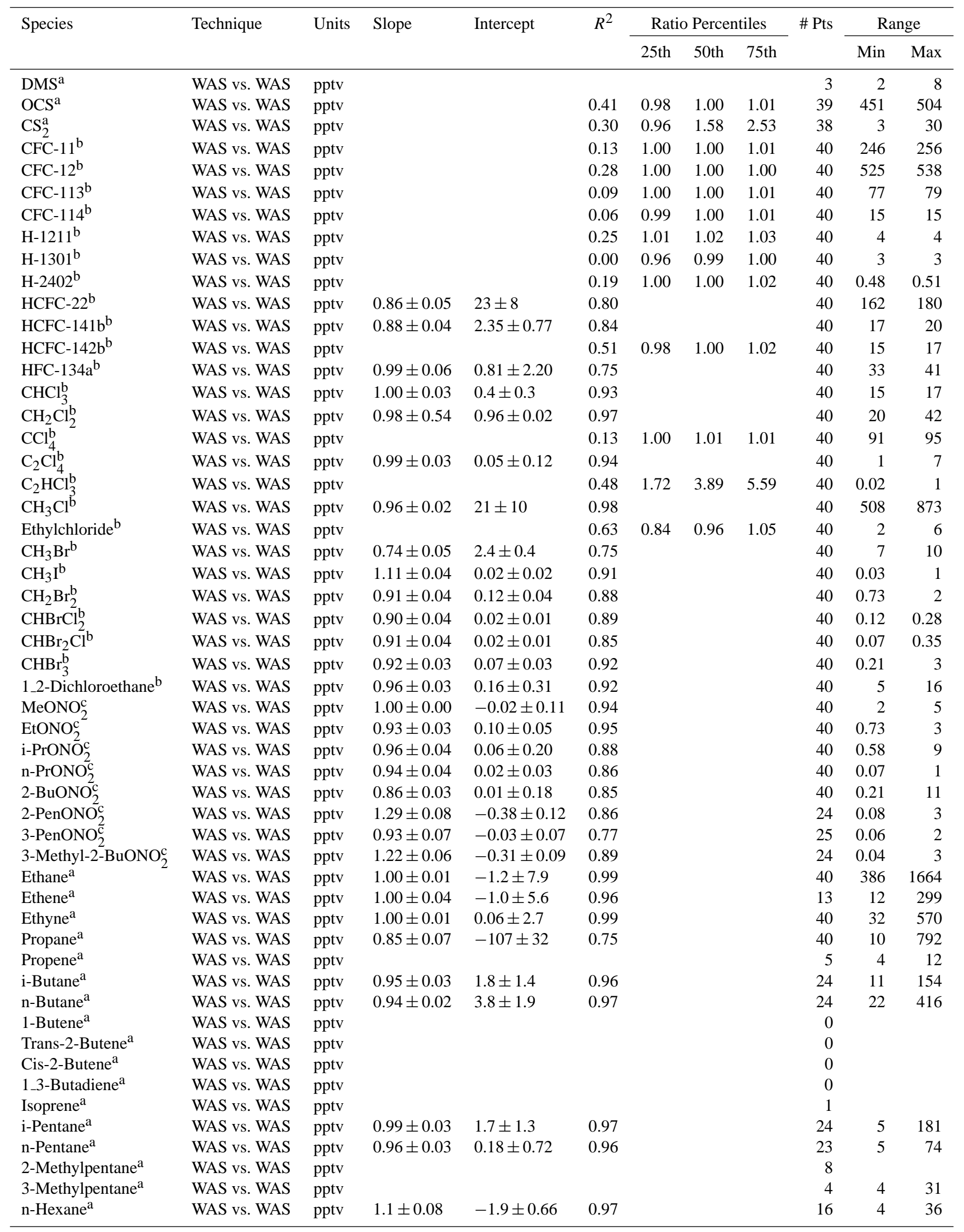

Atmos. Meas. Tech., 4, 9-27, 2011 
Table 3e. Continued.

\begin{tabular}{|c|c|c|c|c|c|c|c|c|c|c|c|}
\hline \multirow[t]{2}{*}{ Species } & \multirow[t]{2}{*}{ Technique } & \multirow[t]{2}{*}{ Units } & \multirow[t]{2}{*}{ Slope } & \multirow[t]{2}{*}{ Intercept } & \multirow[t]{2}{*}{$R^{2}$} & \multicolumn{3}{|c|}{ Ratio Percentiles } & \multirow[t]{2}{*}{ \# Pts } & \multicolumn{2}{|c|}{ Range } \\
\hline & & & & & & 25 th & 50 th & 75th & & Min & Max \\
\hline n-Heptane $\mathrm{a}^{\mathrm{a}}$ & WAS vs. WAS & pptv & & & & & & & 1 & & \\
\hline Benzene $^{a}$ & WAS vs. WAS & pptv & $0.98 \pm 0.01$ & $-0.29 \pm 0.78$ & 0.99 & & & & 36 & 4 & 138 \\
\hline 1_2_4-Trimethylbenzene ${ }^{\mathrm{a}}$ & WAS vs. WAS & pptv & & & & & & & 0 & & \\
\hline 1_3_5-Trimethylbenzene $\mathrm{a}^{\mathrm{a}}$ & WAS vs. WAS & pptv & & & & & & & 0 & & \\
\hline Ethylbenzene $^{a}$ & WAS vs. WAS & pptv & & & & & & & 3 & 4 & 17 \\
\hline i-Propylbenzene ${ }^{a}$ & WAS vs. WAS & pptv & & & & & & & 0 & & \\
\hline n-Propylbenzene ${ }^{a}$ & WAS vs. WAS & pptv & & & & & & & 0 & & \\
\hline Toluene $^{\mathrm{a}}$ & WAS vs. WAS & pptv & $0.93 \pm 0.03$ & $0.16 \pm 1.1$ & 0.98 & & & & 21 & 4 & 151 \\
\hline 3-Ethyltoluene ${ }^{\mathrm{a}}$ & WAS vs. WAS & pptv & & & & & & & 0 & & \\
\hline 4-Ethyltoluene ${ }^{\mathrm{a}}$ & WAS vs. WAS & pptv & & & & & & & 0 & & \\
\hline $\mathrm{m}-X y l e n \mathrm{e}^{\mathrm{a}}$ & WAS vs. WAS & pptv & & & & & & & 0 & & \\
\hline p-Xylene ${ }^{a}$ & WAS vs. WAS & pptv & & & & & & & 0 & & \\
\hline o-Xylene ${ }^{a}$ & WAS vs. WAS & pptv & & & & & & & 1 & & \\
\hline
\end{tabular}

${ }^{a}$ Online files found in VOCs link at http://www-air.larc.nasa.gov/missions/intex-b/intexb.html under the Measurement Comparisons: MILAGRO/INTEX-B/IMPEX link.

$\mathrm{b}$ Online files found in halocarbons link at http://www-air.larc.nasa.gov/missions/intex-b/intexb.html under the Measurement Comparisons: MILAGRO/INTEX-B/IMPEX link.

${ }^{\mathrm{c}}$ Online files found in alkyl nitrates link at http://www-air.larc.nasa.gov/missions/intex-b/intexb.html under the Measurement Comparisons: MILAGRO/INTEX-B/IMPEX link.

Table 3f. Statistical results of DC-8/C-130 intercomparison. $j$-values. NOTE: Technique is listed as X (C-130) vs. Y (DC-8).

\begin{tabular}{|c|c|c|c|c|c|c|c|c|c|c|c|}
\hline \multirow[t]{2}{*}{ Species } & \multirow[t]{2}{*}{ Technique } & \multirow[t]{2}{*}{ Units } & \multirow[t]{2}{*}{ Slope } & \multirow[t]{2}{*}{ Intercept } & \multirow[t]{2}{*}{$R^{2}$} & \multicolumn{3}{|c|}{ Ratio Percentiles } & \multirow[t]{2}{*}{ \# Pts } & \multicolumn{2}{|c|}{ Range } \\
\hline & & & & & & 25 th & 50 th & 75th & & Min & $\operatorname{Max}$ \\
\hline$j\left(\mathrm{O}_{3}\right)$ & SAFS vs. SAFS & $\mathrm{s}^{-1}$ & $1.01 \pm 0.01$ & $0.00 \pm 0.00$ & 0.98 & & & & 850 & $2 \mathrm{E}-5$ & $6 \mathrm{E}-5$ \\
\hline$j\left(\mathrm{NO}_{2}\right)$ & SAFS vs. SAFS & $\mathrm{s}^{-1}$ & $0.93 \pm 0.01$ & $0.00 \pm 0.00$ & 0.98 & & & & 867 & 0.009 & 0.015 \\
\hline
\end{tabular}

estimates were similar; ODR variance and mse were smaller than that from OLS about $25 \%$ of the time. OLS results were significantly better than ODR only $2 \%$ of the time, (Boggs et al., 1988). While ODR allows for the possibility of assigning specific uncertainties to each data point, an accurate estimate of measurement uncertainty is not often available on point by point basis. Even when available, this can be complicated when merging measurements of differing integration times. Therefore, in the interest of treating all the intercomparisons uniformly, we use $w_{\varepsilon_{i}}$ and $w_{\delta_{i}}=1$. The coefficient of determination, $R^{2}$, is used to indicate the quality of the linear relationship between the paired measurements.

\section{Result}

\subsection{INTEX-B intercomparison}

Three types of comparisons were conducted and are presented below: DC-8 to C-130 (Table 3), DC-8 to DC-8 (Table 4), and C-130 to C-130 (Table 5). One hundred and forty parameters were grouped according to chemical similarities and compared. The chemical groups for intercomparison purposes are photochemical precursors and gas phase trac- ers, photochemical products, photochemical radicals, oxygenated volatile organic carbons (OVOCs), non-methane hydrocarbons (NMHCs) along with halocarbons, alkylnitrates, and organic sulfur compounds, photolysis frequencies, particle microphysical properties, particle chemical composition, and particle scattering and absorption.

As stated previously, when $R^{2}$ is greater than or equal to 0.75 , the slope and intercept of the regression are given to represent the level of measurement consistency. It is noted here that the intercept should not simply interpreted as the offset between the instruments. When $R^{2}$ is less than 0.75 percentile statistics are given based on the ratio of the data (DC-8/C-130). The resulting statistics are given in the following Table $3 \mathrm{a}$ through Table $3 \mathrm{i}$ for the DC- 8 to C-130 comparison. All analyses are based on the archived final data combined from all three intercomparison flights. No statistical analyses are provided when there are an insufficient number of data points to adequately represent the entire intercomparison periods. Finally, the range (minimum and maximum) is provided as additional information for the reader. In addition to the comparisons listed in Tables 3, 4, and 5, the uncertainties for each instrument can be found in Table 2. The uncertainties were provided in the final data file archive (Current Archive Status link) 
Table 3g. Statistical results of DC-8/C-130 intercomparison. Particle microphysical properties. NOTE: Technique is listed as X (C-130) vs. Y (DC-8).

\begin{tabular}{|c|c|c|c|c|c|c|c|c|c|c|c|}
\hline \multirow[t]{2}{*}{ Species } & \multirow[t]{2}{*}{ Technique } & \multirow[t]{2}{*}{ Units } & \multirow[t]{2}{*}{ Slope } & \multirow[t]{2}{*}{ Intercept } & \multirow[t]{2}{*}{$R^{2}$} & \multicolumn{3}{|c|}{ Ratio Percentiles } & \multirow[t]{2}{*}{ \# Pts } & \multicolumn{2}{|c|}{ Range } \\
\hline & & & & & & 25 th & 50th & 75th & & Min & Max \\
\hline$N>3 \mathrm{~nm}$ & $\mathrm{CPC}$ & $\mathrm{cm}^{-3}$ & $1.19 \pm 0.00$ & $-188 \pm 36$ & 0.93 & & & & 7908 & 35 & 99831 \\
\hline$N>10 \mathrm{~nm}(05 / 15)$ & $\mathrm{CPC}$ & $\mathrm{cm}^{-3}$ & $0.98 \pm 0.00$ & $0.73 \pm 2.6$ & 0.98 & & & & 2981 & 208 & 3113 \\
\hline$N>10 \mathrm{~nm}(04 / 17)$ & $\mathrm{CPC}$ & $\mathrm{cm}^{-3}$ & $2.18 \pm 0.01$ & $-191 \pm 7$ & 0.94 & & & & 2623 & 119 & 4161 \\
\hline Hot CN (03/19) & $\mathrm{CPC}$ & $\mathrm{cm}^{-3}$ & $0.47 \pm 0.0$ & $871 \pm 17$ & 0.96 & & & & 2290 & 1166 & 24823 \\
\hline Hot CN $(05 / 15)$ & $\mathrm{CPC}$ & $\mathrm{cm}^{-3}$ & $0.94 \pm 0.00$ & $-19 \pm 2$ & 0.98 & & & & 3003 & 70 & 2842 \\
\hline N_DMA & DMA & $\mathrm{cm}^{-3}$ & & & & & & & 11 & & \\
\hline N_OPC & OPC & $\mathrm{cm}^{-3}$ & $0.85 \pm 0.01$ & $0 \pm 0$ & 0.98 & & & & 149 & 4 & 886 \\
\hline N_APS & APS & $\mathrm{cm}^{-3}$ & $1.81 \pm 0.01$ & $-0.14 \pm 0.02$ & 0.97 & & & & 521 & 0.14 & 8 \\
\hline Nsub & OPC & $\mathrm{cm}^{-3}$ & $0.85 \pm 0.01$ & $0 \pm 0$ & 0.98 & & & & 149 & 4 & 884 \\
\hline Nsuper & OPC & $\mathrm{cm}^{-3}$ & $1.29 \pm 0.03$ & $-0.05 \pm 0.02$ & 0.93 & & & & 149 & 0.04 & 2 \\
\hline N_150C_DMA & DMA & $\mathrm{cm}^{-3}$ & & & & & & & 1 & & \\
\hline N_150C_OPC & $\mathrm{OPC}$ & $\mathrm{cm}^{-3}$ & & & & & & & 10 & & \\
\hline Nsub_150C & $\mathrm{OPC}$ & $\mathrm{cm}^{-3}$ & & & & & & & 10 & & \\
\hline Nsuper_150C & $\mathrm{OPC}$ & $\mathrm{cm}^{-3}$ & & & & & & & 10 & & \\
\hline N_300C_DMA & DMA & $\mathrm{cm}^{-3}$ & & & & & & & 1 & & \\
\hline N_300C_OPC & OPC & $\mathrm{cm}^{-3}$ & & & & & & & 5 & & \\
\hline Nsub_300C & $\mathrm{OPC}$ & $\mathrm{cm}^{-3}$ & & & & & & & 5 & & \\
\hline Nsuper_300C & $\mathrm{OPC}$ & $\mathrm{cm}^{-3}$ & & & & & & & 5 & & \\
\hline N_400C_OPC & $\mathrm{OPC}$ & $\mathrm{cm}^{-3}$ & & & & & & & 10 & & \\
\hline Nsub_400C & $\mathrm{OPC}$ & $\mathrm{cm}^{-3}$ & & & & & & & 10 & & \\
\hline Nsuper_400C & $\mathrm{OPC}$ & $\mathrm{cm}^{-3}$ & & & & & & & 10 & & \\
\hline V_DMA & DMA & $\mu \mathrm{m}^{3} \mathrm{~cm}^{-3}$ & & & & & & & 11 & & \\
\hline V_OPC & OPC & $\mu \mathrm{m}^{3} \mathrm{~cm}^{-3}$ & $0.99 \pm 0.01$ & $0.00 \pm 0.05$ & 0.98 & & & & 149 & 0.06 & 9 \\
\hline V_APS & APS & $\mu \mathrm{m}^{3} \mathrm{~cm}^{-3}$ & $2.62 \pm 0.05$ & $-1.4 \pm 0.25$ & 0.83 & & & & 521 & 0.13 & 24 \\
\hline Vsub & $\mathrm{OPC}$ & $\mu \mathrm{m}^{3} \mathrm{~cm}^{-3}$ & $0.92 \pm 0.04$ & $0.0 \pm 0.0$ & 0.98 & & & & 149 & 0.03 & 6 \\
\hline Vsuper & OPC & $\mu \mathrm{m}^{3} \mathrm{~cm}^{-3}$ & $1.14 \pm 0.03$ & $0.0 \pm 0.0$ & 0.81 & & & & 149 & 0.02 & 3 \\
\hline V_150C_DMA & DMA & $\mu \mathrm{m}^{3} \mathrm{~cm}^{-3}$ & & & & & & & 1 & & \\
\hline V_150C_OPC & OPC & $\mu \mathrm{m}^{3} \mathrm{~cm}^{-3}$ & & & & & & & 10 & & \\
\hline Vsub_150C & OPC & $\mu \mathrm{m}^{3} \mathrm{~cm}^{-3}$ & & & & & & & 10 & & \\
\hline Vsuper_150C & OPC & $\mu \mathrm{m}^{3} \mathrm{~cm}^{-3}$ & & & & & & & 10 & & \\
\hline V_300C_DMA & DMA & $\mu \mathrm{m}^{3} \mathrm{~cm}^{-3}$ & & & & & & & 1 & & \\
\hline V_300C_OPC & OPC & $\mu \mathrm{m}^{3} \mathrm{~cm}^{-3}$ & & & & & & & 5 & & \\
\hline Vsub_300C & OPC & $\mu \mathrm{m}^{3} \mathrm{~cm}^{-3}$ & & & & & & & 5 & & \\
\hline Vsuper_300C & $\mathrm{OPC}$ & $\mu \mathrm{m}^{3} \mathrm{~cm}^{-3}$ & & & & & & & 5 & & \\
\hline V_400C_OPC & OPC & $\mu \mathrm{m}^{3} \mathrm{~cm}^{-3}$ & & & & & & & 10 & & \\
\hline Vsub_400C & $\mathrm{OPC}$ & $\mu \mathrm{m}^{3} \mathrm{~cm}^{-3}$ & & & & & & & 10 & & \\
\hline Vsuper_400C & $\mathrm{OPC}$ & $\mu \mathrm{m}^{3} \mathrm{~cm}^{-3}$ & & & & & & & 10 & & \\
\hline
\end{tabular}

Table 3h. Statistical results of DC-8/C-130 intercomparison. Particle chemical composition. NOTE: Technique is listed as X (C-130) vs. Y (DC-8).

\begin{tabular}{|c|c|c|c|c|c|c|c|c|c|c|c|}
\hline \multirow[t]{2}{*}{ Species } & \multirow[t]{2}{*}{ Technique } & \multirow[t]{2}{*}{ Units } & \multirow[t]{2}{*}{ Slope } & \multirow[t]{2}{*}{ Intercept } & \multirow[t]{2}{*}{$R^{2}$} & \multicolumn{3}{|c|}{ Ratio Percentiles } & \multirow[t]{2}{*}{ \# Pts } & \multicolumn{2}{|c|}{ Range } \\
\hline & & & & & & 25 th & 50th & 75th & & Min & Max \\
\hline \multirow[t]{2}{*}{$\mathrm{SO}_{4}^{=\mathrm{a}}$} & MC vs. AMS & $\mu \mathrm{g} \mathrm{m}^{-3}$ & & & 0.37 & 1.03 & 1.49 & 2.02 & 75 & 0.04 & 1.5 \\
\hline & MC vs. PILs & $\mu \mathrm{g} \mathrm{m}^{-3}$ & $0.96 \pm 0.05$ & $-0.07 \pm 0.03$ & 0.89 & & & & 47 & 0.04 & 1.4 \\
\hline
\end{tabular}

\footnotetext{
a Further intercomparisons of the AMS with other instruments during INTEX-B have been presented by DeCarlo et al. (2008) and Dunlea et al. (2009).
} 
Table 3i. Statistical results of DC-8/C-130 intercomparison. Particle scattering and absorption. NOTE: Technique is listed as X (C-130) vs. Y (DC-8).

\begin{tabular}{|c|c|c|c|c|c|c|c|c|c|c|c|}
\hline \multirow[t]{2}{*}{ Species } & \multirow[t]{2}{*}{ Technique } & \multirow[t]{2}{*}{ Units } & \multirow[t]{2}{*}{ Slope } & \multirow[t]{2}{*}{ Intercept } & \multirow[t]{2}{*}{$R^{2}$} & \multicolumn{3}{|c|}{ Ratio Percentiles } & \multirow[t]{2}{*}{ \# Pts } & \multicolumn{2}{|c|}{ Range } \\
\hline & & & & & & 25 th & 50 th & 75 th & & Min & $\operatorname{Max}$ \\
\hline Scatt $450 \mathrm{~nm}$ & TSI Nephelometer & $\mathrm{Mm}^{-1}$ & $1.01 \pm 0.00$ & $-0.18 \pm 0.13$ & 0.99 & & & & 663 & 2 & 113 \\
\hline Scatt $550 \mathrm{~nm}$ & TSI Nephelometer & $\mathrm{Mm}^{-1}$ & $1.08 \pm 0.00$ & $-0.11 \pm 0.10$ & 0.99 & & & & 754 & 0.94 & 83 \\
\hline Scatt $700 \mathrm{~nm}$ & TSI Nephelometer & $\mathrm{Mm}^{-1}$ & $1.11 \pm 0.00$ & $-0.61 \pm 0.07$ & 0.99 & & & & 693 & 1 & 55 \\
\hline Scattsub $550 \mathrm{~nm}$ & RR Nephelometer & $\mathrm{Mm}^{-1}$ & $1.32 \pm 0.01$ & $-0.60 \pm 0.11$ & 0.99 & & & & 652 & 0.23 & 67 \\
\hline Abs $470 \mathrm{~nm}$ & PSAP & $\mathrm{Mm}^{-1}$ & $1.09 \pm 0.02$ & $-0.02 \pm 0.05$ & 0.95 & & & & 112 & 0.04 & 6 \\
\hline Abs $530 \mathrm{~nm}$ & PSAP & $\mathrm{Mm}^{-1}$ & $1.09 \pm 0.03$ & $-0.04 \pm 0.04$ & 0.94 & & & & 110 & 0.03 & 5 \\
\hline Abs $660 \mathrm{~nm}$ & PSAP & $\mathrm{Mm}^{-1}$ & $1.19 \pm 0.03$ & $-0.08 \pm 0.04$ & 0.91 & & & & 98 & 0.02 & 4 \\
\hline SSA & N/A & N/A & & & 0.27 & 0.99 & 1.00 & 1.01 & 104 & 0.83 & 0.98 \\
\hline
\end{tabular}

Table 4a. DC-8 intra-platform comparison. Photochemical precursors. NOTE: Technique is listed as X vs. Y.

\begin{tabular}{|c|c|c|c|c|c|c|c|c|c|c|c|}
\hline \multirow[t]{2}{*}{ Species } & \multirow[t]{2}{*}{ Technique } & \multirow[t]{2}{*}{ Units } & \multirow[t]{2}{*}{ Slope } & \multirow[t]{2}{*}{ Intercept } & \multirow[t]{2}{*}{$R^{2}$} & \multicolumn{3}{|c|}{ Ratio Percentiles } & \multirow[t]{2}{*}{ \# Pts } & \multicolumn{2}{|c|}{ Range } \\
\hline & & & & & & 25 th & 50th & 75th & & Min & $\operatorname{Max}$ \\
\hline $\mathrm{H}_{2} \mathrm{O}$ & DLH vs. Cryo & $\mathrm{g} \mathrm{kg}^{-1}$ & $1.04 \pm 0.00$ & $-0.07 \pm 0.00$ & 0.99 & & & & 8133 & 0.003 & 17 \\
\hline
\end{tabular}

online at the INTEX-B website (http://www-air.larc.nasa. gov/missions/intex-b/intexb.html). For cases where uncertainties were available on a point by point basis, the uncertainty was calculated as a percentage of the measurement. The minimum and maximum percentages are given in parentheses and the median is listed outside the parentheses. We present these comparisons and uncertainties without rating the level of agreement. This is a highly subjective task and we leave it to the reader to make that judgment with appropriate consultation with the respective PIs. For an explanation of "Technique", the reader is referred to the individual PI files located on the INTEX-B website (http: //www-air.larc.nasa.gov/missions/intex-b/intexb.html) under the Current Archive Status link.

All intercomparison correlation plots can be found online under the Measurement Comparisons: MILAGRO/INTEXB/IMPEX link at http://www-air.larc.nasa.gov/missions/ intex-b/intexb.html. The correlation of the combined the data from all three flights is in the summary section. Individual timeseries and correlation plots are also available for each intercomparison on 19 March, 17 April, and 15 May 2006.

As described earlier, intra-platform comparisons were also conducted on both the DC-8 and C-130 aircraft for any overlapping measurements. See Table $4 \mathrm{a}$ through Table $4 \mathrm{c}$ for a complete list of the species, techniques used, and a statistical summary for the DC- 8 to DC- 8 comparisons. Tables 5ae provide statistical summary for the C-130 to C-130 comparisons. Since the instruments were located on the same platform, comparison data was not limited to the intercomparison portions of the flights. Data from the entire mission could be included.

\subsection{Comparison with ICARTT data}

In addition to the intercomparisons made during INTEX$\mathrm{B}$, we wish to examine the cases where the same comparisons could be made with data from the ICARTT mission and highlight instances where those intercomparisons show significant change. The ICARTT mission was conducted in 2004, a portion of which was INTEX-A (the predecessor to INTEX-B). For a complete description of INTEX-A see Singh et al. (2006). A full listing of the INTEX-A intercomparisons can be found at http://www-air.larc.nasa.gov/ missions/intexna/meas-comparison.htm . There are three cases where significant change is observed between INTEXA and INTEX-B; $\mathrm{H}_{2} \mathrm{O}_{2}, \mathrm{PAN}$, and total PANs. For $\mathrm{H}_{2} \mathrm{O}_{2}$ the comparison was a DC-8 intra-platform comparison between CIT CIMS and URI EFD during INTEX-A (Fig. 4a) while for INTEX-B, CIT CIMS was on the C-130 and URI EFD on the DC-8 (Fig. 4b). The INTEX-A comparison included significantly more data pairs and covered a wider range of values since both instruments were on the same aircraft and all mission data could be used. During INTEX-B, $R^{2}$ is much improved (0.92 in INTEX-B vs. 0.77 during INTEX-A) however the slope of the regression was better during INTEX-A (1.01 for INTEX-A vs. 1.24 for INTEX-B). This could be due to the smaller amount of data during INTEX-B as well as the smaller dynamic range for the INTEX-B intercomparison measurements.

For PAN, the same instruments were used for both missions ARC PANAK (or dual GC) on the DC-8 for both INTEX-A and INTEX-B; NCAR CIGAR on the NOAA WP-3D for INTEX-A and on the C-130 for INTEX-B). In 
Table 4b. DC-8 intra-platform comparison. Photochemical products. NOTE: Technique is listed as X vs. Y.

\begin{tabular}{|c|c|c|c|c|c|c|c|c|c|c|c|}
\hline \multirow[t]{2}{*}{ Species } & \multirow[t]{2}{*}{ Technique } & \multirow[t]{2}{*}{ Units } & \multirow[t]{2}{*}{ Slope } & \multirow[t]{2}{*}{ Intercept } & \multirow[t]{2}{*}{$R^{2}$} & \multicolumn{3}{|c|}{ Ratio Percentiles } & \multirow[t]{2}{*}{ \# Pts } & \multicolumn{2}{|c|}{ Range } \\
\hline & & & & & & 25 th & 50 th & 75th & & Min & $\operatorname{Max}$ \\
\hline $\mathrm{CH}_{2} \mathrm{O}$ & TDL vs. EFD & pptv & $0.83 \pm 0.01$ & $-12 \pm 8$ & 0.88 & & & & 2119 & LOD & 18830 \\
\hline $\mathrm{H}_{2} \mathrm{O}_{2}$ & ACCD vs. EFD & pptv & & & 0.67 & 0.56 & 0.80 & 1.07 & 1962 & 27 & 9899 \\
\hline $\mathrm{HNO}_{3}$ & TDLIF vs. MC & pptv & $0.91 \pm 0.01$ & $-28 \pm 4$ & 0.84 & & & & 2270 & 3 & 7530 \\
\hline
\end{tabular}

Table 4c. DC-8 intra-platform comparison. $j$-values. NOTE: Technique is listed as X vs. Y.

\begin{tabular}{|c|c|c|c|c|c|c|c|c|c|c|c|}
\hline \multirow[t]{2}{*}{ Species } & \multirow[t]{2}{*}{ Technique } & \multirow[t]{2}{*}{ Units } & \multirow[t]{2}{*}{ Slope } & \multirow[t]{2}{*}{ Intercept } & \multirow{2}{*}{$R^{2}$} & \multicolumn{3}{|c|}{ Ratio Percentiles } & \multirow[t]{2}{*}{ \# Pts } & \multicolumn{2}{|c|}{ Range } \\
\hline & & & & & & 25 th & 50 th & 75th & & Min & $\operatorname{Max}$ \\
\hline$j\left(\mathrm{NO}_{2}\right)$ & SAFS vs. Filt. Rad. & $\mathrm{s}^{-1}$ & $0.96 \pm 0.00$ & $0.00 \pm 0.00$ & 0.99 & & & & 6846 & LOD & 0.02 \\
\hline
\end{tabular}

Table 5a. C-130 intra-platform comparison. Gas phase tracers. NOTE: Technique is listed as X vs. Y.

\begin{tabular}{|c|c|c|c|c|c|c|c|c|c|c|c|}
\hline \multirow[t]{2}{*}{ Species } & \multirow[t]{2}{*}{ Technique } & \multirow[t]{2}{*}{ Units } & \multirow[t]{2}{*}{ Slope } & \multirow[t]{2}{*}{ Intercept } & \multirow[t]{2}{*}{$R^{2}$} & \multicolumn{3}{|c|}{ Ratio Percentiles } & \multirow[t]{2}{*}{ \# Pts } & \multicolumn{2}{|c|}{ Range } \\
\hline & & & & & & 25 th & 50 th & 75 th & & Min & $\operatorname{Max}$ \\
\hline $\mathrm{SO}_{2}$ & CIMS vs. UVF & ppbv & $0.76 \pm 0.00$ & $0.24 \pm 0.03$ & 0.90 & & & & 5799 & LOD & 392 \\
\hline $\mathrm{SO}_{2}$ & CIMS vs. UVF ${ }^{b}$ & ppbv & $0.87 \pm 0.00$ & $0.07 \pm 0.02$ & 0.91 & & & & 5854 & LOD & 100 \\
\hline $\mathrm{CH}_{3} \mathrm{CN}$ & PTRMS vs. TOGA & pptv & & & 0.40 & 0.71 & 0.96 & 1.33 & 1575 & LOD & 5.13 \\
\hline
\end{tabular}

${ }^{\text {a }}$ All data. ${ }^{\mathrm{b}} \mathrm{SO}_{2} \leq 100$ ppbv.

Table 5b. C-130 intra-platform comparison. Photochemical products. NOTE: Technique is listed as X vs. Y.

\begin{tabular}{|c|c|c|c|c|c|c|c|c|c|c|c|}
\hline \multirow[t]{2}{*}{ Species } & \multirow[t]{2}{*}{ Technique } & \multirow[t]{2}{*}{ Units } & \multirow[t]{2}{*}{ Slope } & \multirow[t]{2}{*}{ Intercept } & \multirow[t]{2}{*}{$R^{2}$} & \multicolumn{3}{|c|}{ Ratio Percentiles } & \multirow[t]{2}{*}{ \# Pts } & \multicolumn{2}{|c|}{ Range } \\
\hline & & & & & & 25 th & 50 th & 75th & & Min & $\operatorname{Max}$ \\
\hline Acetic Acid & CIMS vs. PTRMS & pptv & & & 0.55 & 0.40 & 0.76 & 1.36 & 3909 & LOD & 10 \\
\hline
\end{tabular}

Table 5c. C-130 intra-platform comparison. Oxygenated volatile organic carbons. NOTE: Technique is listed as X vs. Y.

\begin{tabular}{|c|c|c|c|c|c|c|c|c|c|c|c|}
\hline \multirow[t]{2}{*}{ Species } & \multirow[t]{2}{*}{ Technique } & \multirow[t]{2}{*}{ Units } & \multirow[t]{2}{*}{ Slope } & \multirow[t]{2}{*}{ Intercept } & \multirow[t]{2}{*}{$R^{2}$} & \multicolumn{3}{|c|}{ Ratio Percentiles } & \multirow[t]{2}{*}{ \# Pts } & \multicolumn{2}{|c|}{ Range } \\
\hline & & & & & & 25 th & 50th & 75 th & & Min & $\operatorname{Max}$ \\
\hline Acetaldehyde & PTRMS vs. TOGA & pptv & & & 0.50 & 0.68 & 1.24 & 2.58 & 1511 & LOD & 11.3 \\
\hline Methanol & PTRMS vs. TOGA & pptv & & & 0.72 & 0.56 & 0.83 & 1.24 & 3442 & 0.02 & 37 \\
\hline
\end{tabular}

this case, the INTEX-A intercomparison was better than the INTEX-B intercomparison. During INTEX-B, $R^{2}=0.77$ and slope $=1.68$, while for INTEX-A $R^{2}=0.82$ and slope $=0.99$. During INTEX-B most data was below 500 pptv (19 March flight had values up to about $1400 \mathrm{pptv}$ ). For INTEX-A most data was also below 500 pptv with a few points up to about 750 pptv. During INTEX-B the higher values skewed the regression slope. Removing the 5 points where either the DC- 8 or C- 130 value is above 500 pptv increases $R^{2}$ slightly to 0.79 and decreases the slope to 1.23 .

The total PANs intercomparisons for INTEX-A and INTEX-B included the same instruments for both missions, with instruments on separate planes for both missions. Both intercomparisons are generally consistent (INTEX-B 

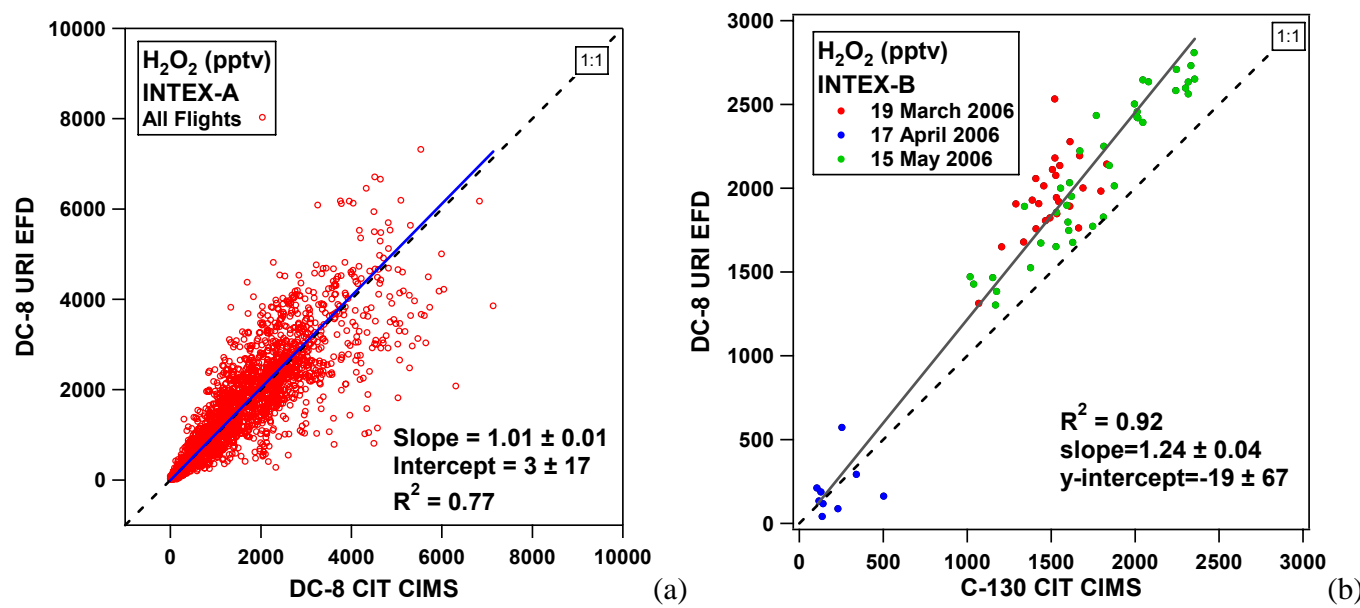

Fig. 4. (a) Scatter plot and orthogonal distance regression for the DC-8 CIMS and EFD $\mathrm{H}_{2} \mathrm{O}_{2}$ intercomparison of all INTEX-A flights. (b) Scatter plot and orthogonal distance regression for the DC-8 and C-130 $\mathrm{H}_{2} \mathrm{O}_{2}$ INTEX-B intercomparisons on 19 March (red), 17 April (blue), and 15 May (green) 2006.

Table 5d. C-130 intra-platform comparison. Nonmethane hydrocarbons, halocarbons, and organic sulfur compounds. NOTE: Technique is listed as X vs. Y.

\begin{tabular}{|c|c|c|c|c|c|c|c|c|c|c|c|}
\hline \multirow[t]{2}{*}{ Species } & \multirow[t]{2}{*}{ Technique } & \multirow[t]{2}{*}{ Units } & \multirow[t]{2}{*}{ Slope } & \multirow[t]{2}{*}{ Intercept } & \multirow[t]{2}{*}{$R^{2}$} & \multicolumn{3}{|c|}{ Ratio Percentiles } & \multirow[t]{2}{*}{ \# Pts } & \multicolumn{2}{|c|}{ Range } \\
\hline & & & & & & 25 th & 50th & 75th & & Min & $\operatorname{Max}$ \\
\hline DMS & TOGA vs. WAS & pptv & & & & & & & 44 & & \\
\hline $\mathrm{CHCl}_{3}^{\mathrm{a}}$ & TOGA vs. WAS & pptv & $1.25 \pm 0.03$ & $0.20 \pm 0.22$ & 0.86 & & & & 388 & 5 & 14 \\
\hline $\mathrm{CHCl}_{3}^{b}$ & TOGA vs. WAS & pptv & & & 0.47 & 0.74 & 0.79 & 0.85 & 256 & 5 & 17 \\
\hline $\mathrm{CH}_{3} \mathrm{Cl}$ & TOGA vs. WAS & pptv & & & 0.02 & 0.96 & 1.05 & 1.11 & 287 & 281 & 1509 \\
\hline i-Butane & TOGA vs. WAS & pptv & $1.06 \pm 0.01$ & $0.62 \pm 3.35$ & 0.93 & & & & 455 & 2 & 608 \\
\hline n-Butane & TOGA vs. WAS & pptv & $0.85 \pm 0.01$ & $22.3 \pm 7.3$ & 0.94 & & & & 571 & 4 & 1634 \\
\hline i-Pentane & TOGA vs. WAS & pptv & $1.19 \pm 0.01$ & $13.3 \pm 3.1$ & 0.95 & & & & 523 & 1 & 938 \\
\hline n-Pentane & TOGA vs. WAS & pptv & $0.87 \pm 0.01$ & $4 \pm 2$ & 0.93 & & & & 471 & 2 & 436 \\
\hline Isoprene & TOGA vs. WAS & pptv & & & & & & & 1 & & \\
\hline Benzene & TOGA vs. WAS & pptv & $1.26 \pm 0.02$ & $-16.4 \pm 1.7$ & 0.91 & & & & 664 & 8 & 336 \\
\hline Toluene & TOGA vs. WAS & pptv & $1.19 \pm 0.02$ & $1.7 \pm 9.1$ & 0.79 & & & & 440 & 0.44 & 1112 \\
\hline o-Xylene & TOGA vs. WAS & pptv & & & & & & & 91 & & \\
\hline
\end{tabular}

${ }^{a}$ Pacific Phase.

b Mexico City Phase.

$R^{2}=0.94$, slope $=1.35 ;$ INTEX-A $R^{2}=0.87$, slope $=0.95$ ). $R^{2}$ was better for INTEX-B while the slope of the regression was better for INTEX-A. The range of values during INTEX$\mathrm{B}$ is almost twice the range during INTEX-A. Again, during INTEX-B a few high values from the 19 March flight skew the slope of the regression. By removing the seven points above $1000 \mathrm{pptv}$, the slope is reduced to $1.15,\left(R^{2}\right.$ is also reduced to a value of 0.84 ).

\section{Summary}

This paper provides a comprehensive overview and a record of measurement consistency of approximately 140 intercomparisons of data acquired during the INTEX-B airborne field campaign conducted in the spring of 2006. A complete set of timeseries and correlation figures can be found at http://www-air.larc.nasa.gov/missions/intex-b/intexb.html under the Measurement Comparisons: MILAGRO/INTEXB/IMPEX link. For interpretation and most effective use of these results, the reader is strongly urged to consult with the instrument PIs. We leave it to the reader to determine the level of consistency between the instruments compared. This should be done not only with the statistical analyses provided in Tables 3, 4, and 5, but also in consideration of the uncertainties in Table 2, keeping in mind that even when measurements are technically consistent within the PI reported uncertainties, significant differences between the measurements can still exist if the uncertainties are large. In addition, future instrument work may benefit from this assessment. 
Table 5e C-130 intra-platform comparison. Particle chemical composition. NOTE: Technique is listed as X vs. Y.

\begin{tabular}{|c|c|c|c|c|c|c|c|c|c|c|c|}
\hline \multirow[t]{2}{*}{ Species } & \multirow[t]{2}{*}{ Technique } & \multirow[t]{2}{*}{ Units } & \multirow[t]{2}{*}{ Slope } & \multirow[t]{2}{*}{ Intercept } & \multirow[t]{2}{*}{$R^{2}$} & \multicolumn{3}{|c|}{ Ratio Percentiles } & \multirow[t]{2}{*}{ \# Pts } & \multicolumn{2}{|c|}{ Range } \\
\hline & & & & & & 25 th & 50th & 75th & & Min & Max \\
\hline $\mathrm{SO}_{4}^{=\mathrm{a}}$ & PILS vs. AMS & $\mu \mathrm{g} \mathrm{m}^{-3}$ & & & 0.45 & 0.50 & 0.88 & 1.50 & 3669 & 0.02 & 15.8 \\
\hline $\mathrm{NO}_{3}^{-\mathrm{a}}$ & PILS vs. AMS & $\mu \mathrm{g} \mathrm{m}^{-3}$ & $1.54 \pm 0.03$ & $0.15 \pm 0.10$ & 0.88 & & & & 410 & 0.02 & 25 \\
\hline $\mathrm{NH}_{4}^{+\mathrm{a}}$ & PILS vs. AMS & $\mu \mathrm{g} \mathrm{m}^{-3}$ & $0.78 \pm 0.01$ & $0.02 \pm 0.02$ & 0.75 & & & & 2496 & 0.1 & 9.4 \\
\hline
\end{tabular}

a Further intercomparisons of the AMS with other instruments during INTEX-B have been presented by DeCarlo et al. (2008) and Dunlea et al. (2009).

\section{Appendix A}

Acronyms and abbreviations.

Abs $470 \mathrm{~nm} \quad$ Aerosol absorption coefficient at $470 \mathrm{~nm}$

Abs $530 \mathrm{~nm} \quad$ Aerosol absorption coefficient at $530 \mathrm{~nm}$

Abs $660 \mathrm{~nm}$ Aerosol absorption coefficient at $660 \mathrm{~nm}$

ACCD Aqueous Collection Chemiluminescence Detection

ACD Atmospheric Chemistry Division

AMS Aerodyne High-Resolution Aerosol Mass Spectrometer

APS Aerodynamic Particle Sizer

ARC Ames Research Center

ARIM Atmospheric Radiation Investigation and Measurements

ATHOS Airborne Tropospheric Hydrogen Oxides Sensor

CIGAR CIMS Instrument by Georgia Tech and NCAR

CIMS Chemical Ionization Mass Spectrometry

CIT California Institute of Technology

CITE Chemical Instrumentation Test and Evaluation

CLD Chemiluminescence Detector

$\mathrm{CN} \quad$ Condensation nuclei

CPC Condensation Particle Counter

Cryo Cryo-hygrometer

DACOM Differential Absorption CO Measurement

DFG Difference Frequency Generation Absorption Spectrometer

DLH Diode Laser Hygrometer

DMA Differential Mobility Analyzer

DMS

EFD

FT

GIT

$\mathrm{HCN}$

Dimethyl sulfide

Enzyme Fluorescence Detection

Free troposphere

Georgia Institute of Technology

Hydrogen cyanide
Hot CN

ICARTT

IMPEX

INTEX-A

INTEX-B

INTEX-NA

ITOP

LaRC

LOD

$\mathrm{MC}$

MCWG

MEK

MILAGRO

MBL

N_150C_DMA

Condensation nuclei with heated inlet to $300^{\circ} \mathrm{C}$

International Consortium for Atmospheric Research on Transport and Transformation

Intercontinental and Mega-city Pollution Experiment

Intercontinental Chemical and Transport Experiment - A

Intercontinental Chemical and Transport Experiment - B

Intercontinental Chemical and Transport Experiment - North America

Intercontinental Transport of Ozone and Precursors

Langley Research Center

Limit of Detection

Mist Chamber

Measurement Comparison Working Group

Methyl ethyl ketone

Mega-city Initiative: Local and Global

Research Observations

Marine boundary layer

Aerosol number density, inlet heated to

$150^{\circ} \mathrm{C}$, measured with differential mobility analyzer

N_150C_OPC Aerosol number density, inlet heated to $150^{\circ} \mathrm{C}$, measured with optical particle counter

N_300C_DMA Aerosol number density, inlet heated to $300^{\circ} \mathrm{C}$, measured with differential mobility analyzer

N_300C_OPC Aerosol number density, inlet heated to $300^{\circ} \mathrm{C}$, measured with optical particle counter

N_400C_OPC Aerosol number density, inlet heated to $400^{\circ} \mathrm{C}$, measured with optical particle counter

N_APS Aerosol number density, measured with aerodynamic particle sizer

N_DMA Aerosol number density, measured with differential mobility analyzer 
N_OPC

NCAR

NEAQS - ITCT 2004

NMHCs

NOAA

$\mathrm{NO}_{\mathrm{y}}$

NSERC

NSF

Nsub

Nsub_150C

Nsub_300C

Nsub_400C

Nsuper

Nsuper_150C

Nsuper_300C

Nsuper_400C

ODR

OLS

OPC

OVOC

PAN

PANAK

PILS

PSAP

PTRMS

RAF

RR Nephelometer

SAFS

Scatt $450 \mathrm{~nm}$

Scatt $550 \mathrm{~nm}$
Aerosol number density, measured with optical particle counter

National Aeronautics and Space Administration

National Center for Atmospheric Research

New England Air Quality Study

- Intercontinental Transport and

Chemical Transformation, 2004

Non-methane hydrocarbons

National Oceanic and Atmospheric Administration

Reactive nitrogen

National Suborbital Education

and Research Center

National Science Foundation

Submicron aerosol number den-

sity

Submicron aerosol number den-

sity, inlet heated to $150^{\circ} \mathrm{C}$

Submicron aerosol number density, inlet heated to $300^{\circ} \mathrm{C}$

Submicron aerosol number density, inlet heated to $400{ }^{\circ} \mathrm{C}$

Supermicron aerosol number density

Supermicron aerosol number density, inlet heated to $150{ }^{\circ} \mathrm{C}$

Supermicron aerosol number density, inlet heated to $300^{\circ} \mathrm{C}$

Supermicron aerosol number density, inlet heated to $400{ }^{\circ} \mathrm{C}$

Orthogonal Distance Regression

Ordinary Least Squares

Optical Particle Counter

Oxygenated Volatile Organic Carbon

Peroxyacetyl Nitrate

PAN/Aldehyde/Ketone Photo Ionization Detector

Particle-Into-Liquid Sampler

Particle Soot Absorption Photometer

Proton Transfer Reaction Mass

Spectrometry

Research Aviation Facility

Radiance Research nephelometer

Scanning actinic flux spectroradiometer

Aerosol scattering coefficient at $450 \mathrm{~nm}$

Aerosol scattering coefficient at $550 \mathrm{~nm}$
Scatt $700 \mathrm{~nm}$

Scattsub $550 \mathrm{~nm}$

SSA

TD-LIF

TDL

TOGA

TRACE-P

TSI Nephelometer

UC

UCI

UND

UNH

URI

USNA

V_APS

V_DMA

V_OPC

V_150C_DMA

V_150C_OPC

V_300C_DMA

V_300C_OPC

V_400C_OPC

UVF

Vsub

Vsub_150C

Vsub_300C

Vsub_400C

Vsuper
Aerosol scattering coefficient at $700 \mathrm{~nm}$

Submicron aerosol scattering coefficient at $550 \mathrm{~nm}$

Single Scattering Albedo

Thermal dissociation-laser induced fluorescence

Tunable Diode Laser Absorption Spectrometer

Trace Organic Gas Analyzer

Transport and Chemical Evolution over the Pacific

TSI, Inc. nephelometer

University of California

University of California, Irvine

University of North Dakota

University of New Hampshire

University of Rhode Island

United States Naval Academy

Aerosol volume density, mea-

sured with aerodynamic particle

sizer

Aerosol volume density, measured with differential mobility analyzer

Aerosol volume density, measured with optical particle counter

Aerosol volume density, inlet heated to $150^{\circ} \mathrm{C}$, measured with differential mobility analyzer

Aerosol volume density, inlet heated to $150^{\circ} \mathrm{C}$, measured with optical particle counter

Aerosol volume density, inlet heated to $300^{\circ} \mathrm{C}$, measured with differential mobility analyzer

Aerosol volume density, inlet heated to $300^{\circ} \mathrm{C}$, measured with optical particle counter

Aerosol volume density, inlet heated to $400^{\circ} \mathrm{C}$, measured with optical particle counter

Ultra-violet fluorescence

Submicron aerosol volume density

Submicron aerosol volume density, inlet heated to $150^{\circ} \mathrm{C}$

Submicron aerosol volume density, inlet heated to $300^{\circ} \mathrm{C}$

Submicron aerosol volume density, inlet heated to $400^{\circ} \mathrm{C}$

Supermicron aerosol volume density 

Vsuper_150C
Supermicron aerosol volume density, inlet heated to $150^{\circ} \mathrm{C}$
Vsuper_300C Supermicron aerosol volume density, inlet heated to $300^{\circ} \mathrm{C}$
Vsuper_400C Supermicron aerosol volume density, inlet heated to $400{ }^{\circ} \mathrm{C}$
WAS

Acknowledgements. The authors wish to thank the National Aeronautics and Space Administration (NASA) Tropospheric Chemistry (TCP) and Making Earth System data records for Use in Research Environments (MEaSUREs) Programs for their support of the measurements and intercomparisons presented in this paper. We also thank the National Science Foundation Atmospheric Chemistry Program for support of this study. We would like to thank the pilots and crew of the NASA DC-8 and the NSF C-130 and the INTEX-B and IMPEX/MILAGRO science teams for contributing to the success of this study. Finally, we thank Ms. Amy Thornhill for her assistance in acquiring additional uncertainty information from principal investigators.

Edited by: D. Heard

\section{References}

Bahreini, R., Evans, B., Middlebrook,A. M., Warneke, C., de Gouw, J. A., De Carlo, P. F., Jimenez, J. L., Brock, C. A., Neuman, J. A., Ryerson, T. B., Stark, H., Atlas, E., Brioude, J., Fried, A., Holloway, J. S., Peischl, J., Richter, D., Walega, J., Weibring, P., Wollny, A. G., and Fehsenfeld, F. C.: Organic aerosol formation in urban and industrial plumes near Houston and Dallas, Texas, J. Geophys. Res., 114, DF00F16, doi:10.1029/2008JD011493, 2009.

Beck, S. M., Bendura, R. J., McDougal, D. S., Hoell, J. M., Gregory, G. L., Curfman, H. J., Davis, D. D., Bradshaw, J., Rodgers, M. O., Wang, C. C., Davis, L. I., Campbell, M. J., Torres, A. L., Carroll, M. A., Ridley, B. A., Sachse, G. W., Hill, G. F., Condon, E. P., and Rasmussen, R. A.: Operational Overview of NASA GTE/CITE 1 Airborne Instrument Intercomparisons: Carbon Monoxide, Nitric Oxide, and Hydroxyl Instrumentation, J. Geophys. Res, 92, 1977-1985, 1987.

Boggs, P. T., Spiegelman, C. H., Donaldson, J. R., and Schnabel, R. B.: A computational examination of orthogonal distance regression, J. Econometr., 38, 169-201, 1988.

DeCarlo, P. F., Dunlea, E. J., Kimmel, J. R., Aiken, A. C., Sueper, D., Crounse, J., Wennberg, P. O., Emmons, L., Shinozuka, Y., Clarke, A., Zhou, J., Tomlinson, J., Collins, D. R., Knapp, D., Weinheimer, A. J., Montzka, D. D., Campos, T., and Jimenez, J. L.: Fast airborne aerosol size and chemistry measurements above Mexico City and Central Mexico during the MILAGRO campaign, Atmos. Chem. Phys., 8, 4027-4048, doi:10.5194/acp8-4027-2008, 2008.

Dunlea, E. J., DeCarlo, P. F., Aiken, A. C., Kimmel, J. R., Peltier, R. E., Weber, R. J., Tomlinson, J., Collins, D. R., Shinozuka, Y., McNaughton, C. S., Howell, S. G., Clarke, A. D., Emmons, L. K., Apel, E. C., Pfister, G. G., van Donkelaar, A., Martin, R. V., Millet, D. B., Heald, C. L., and Jimenez, J. L.: Evolution of Asian aerosols during transpacific transport in INTEX-B, At- mos. Chem. Phys., 9, 7257-7287, doi:10.5194/acp-9-7257-2009, 2009.

Eisele, F. L., Mauldin, L., Cantrell, C., Zondlo, M., Apel, E., Fried, A., Walega, J., Shetter, R., Lefer, B., Flocke, F., Weinheimer, A., Avery, M., Vay, S., Sachse, G., Podolske, J., Diskin, G., Barrick, J. D., Singh, H. B., Brune, W., Harder, H., Martinez, M., Bandy, A., Thornton, D., Heikes, B., Kondo, Y., Reimer, D., Sandholm, S., Tan, D., Talbot, R., and Dibb, J.: Summary of measurement intercomparisons during TRACE-P, J. Geophys. Res., 108, 8791, doi:10.1029/2002JD003167, 2003.

Gregory, G. L., Hoell, J. M., Beck, S. M., McDougal, D. S., Meyers, J. A., and Bruton, D. B.: Operational Overview of Wallops Island Instrument Intercomparison: Carbon Monoxide, Nitric Oxide, and Hydroxyl Instrumentation, J. Geophys. Res., 90, 1280812818, 1985.

Gregory, G. L., Davis, D. D., Beltz, N., Bandy, A. R., Ferek, R. J., and Thornton, D. C.: An Intercomparison of Aircraft Instrumentation for Tropospheric Measurements of Sulfur Dioxide, J. Geophys. Res., 98, 23325-23352, 1993 a.

Gregory, G. L., Davis, D. D., Thornton, D. C., Johnson, J. E., Bandy, A. R., Saltzman, E. S., Andreae, M. O., and Barrick, J. D.: An Intercomparison of Aircraft Instrumentation for Tropospheric Measurements of Carbonyl Sulfide, Hydrogen Sulfide, and Carbon Disulfide, J. Geophys. Res., 98, 23353-23372, $1993 b$.

Gregory, G. L., Warren, L. S., Davis, D. D., Andreae, M. O., Bandy, A. R., Ferek, R. J., Johnson, J. E., Saltzman, E. S., and Cooper, D. J.: An Intercomparison of Instrumentation for Tropospheric Measurements of Dimethyl Sulfide: Aircraft Results for Concentrations at the Parts-Per-Trillion Level, J. Geophys. Res., 98, 23373-23388, 1993c.

Hoell, J. M., Gregory, G. L., Carroll, M. A., McFarland, M., Ridley, B. A., Davis, D. D., Bradshaw, J., Rodgers, M. O., Torres, A. L., Sachse, G. W., Hill, G. F., Condon, E. P., Rasmussen, R. A., Campbell, M. C., Farmer, J. C., Sheppard, J. C., Wang, C. C., and Davis, L. I.: An Intercomparison of Carbon Monoxide, Nitric Oxide, and Hydroxyl Measurement Techniques: Overview of Results, J. Geophys. Res., 89, 11819-11825, 1984.

Hoell, J. M., Gregory, G. L., McDougal, D. S., Carroll, M. A., McFarland, M., Ridley, B. A., Davis, D. D., Bradshaw, J., Rodgers, M. O., and Torresm A. L.: An Intercomparison of Nitric Oxide Measurement Techniques, J. Geophys. Res., 90, 12843-12852, 1985a.

Hoell, J. M., Gregory, G. L., McDougal, D. S., Sachse, G. W., Hill, G. F., Condon, E. P., and Rasmussen, R. A.: An Intercomparison of Carbon Monoxide Measurement Techniques, J. Geophys. Res., 90, 12881-12890, 1985 b.

Hoell, J. M., Albritton, D. L., Gregory, G. L., McNeal, R. J., Beck, S. M., Bendura, R. J., and Drewery, J. W.: Operational Overview of NASA GTE/CITE 2 Airborne Instrument Intercomparisons: Nitrogen Dioxide, Nitric Acid, and Peroxyacetyl Nitrate, J. Geophys. Res., 95, 10047-10057, 1990.

Hoell, J. M., Davis, D. D., Gregory, G. L., McNeal, R. J., Bendura, R. J., Drewery, J. W., Barrick, J. D., Kirchhoff, V. W. J. H., Motta, A. G., Navarro, R. L., Dorko, W. D., and Owen, D. W.: Operational Overview of the NASA GTE/CITE 3 Airborne Instrument Intercomparisons for Sulfur Dioxide, Hydrogen Sulfide, Carbonyl Sulfide, Dimethyl Sulfide, and Carbon Disulfide, J. Geophys. Res., 98, 23291-23304, 1993. 
Singh, H. B., Brune, W. H., Crawford, J. H., Jacob, D. J., and Russell, P. B.: Overview of the summer 2004 Intercontinental Chemical Transport Experiment- North America (INTEX-A), J. Geophys. Res., 111, D24S01, doi:10.1029/2006JD007905, 2006.

Singh, H. B., Brune, W. H., Crawford, J. H., Flocke, F., and Jacob, D. J.: Chemistry and transport of pollution over the Gulf of Mexico and the Pacific: spring 2006 INTEX-B campaign overview and first results, Atmos. Chem. Phys., 9, 2301-2318, doi:10.5194/acp-9-2301-2009, 2009.
Zwolak, J. W., Boggs, P. T., and Watson, L. T.: Algorithm 869: ODRPACK95: A weighted orthogonal distance regression code with bound constraints, http://doi.acm.org/10.1145/1268776. 1268782, last access: 3 January 2011, ACM Trans. Math. Softw., 33(4), 27, doi:10.1145/1268776.1268782, 2007. 